\title{
Bi-dimensional element stratifications computed for magnetic Ap star atmospheres ${ }^{\star}$
}

\author{
G. Alecian ${ }^{1}$ and M. J. Stift ${ }^{2,1}$ \\ 1 LUTH, Observatoire de Paris, CNRS, Université Paris Diderot, 5 Place Jules Janssen, 92190 Meudon, France \\ e-mail: georges.alecian@obspm. fr \\ 2 Institut für Astronomie (IfA), Universität Wien, Türkenschanzstrasse 17, 1180 Wien, Austria
}

Received 30 November 2009 / Accepted 2 March 2010

\begin{abstract}
Context. Theoretical modelling of abundance stratifications and surface distributions of chemical elements in Ap stars constitutes a major challenge. The atomic diffusion model provides the most appropriate framework in which to understand these abundance anomalies.

Aims. We present theoretical 2D stratifications of 16 metals in upper main sequence chemically peculiar stars, with and without magnetic fields to provide a reference point for further theoretical and observational studies.

Methods. We used our code CaratStrat to compute a large grid of stratifications (equilibrium solutions in LTE) for plane-parallel $T_{\text {eff }}=8500,10000,12000$, and $14000 \mathrm{~K}$ stellar atmospheres. By interpolation, we constructed bi-dimensional cuts through these stellar atmospheres, which are permeated by a dipolar magnetic field of strength $20 \mathrm{kG}$ at the magnetic pole. We also provide vertical (1D) stratifications of metals in non-magnetic stars $(\mathrm{HgMn})$.

Results. We present a large number of 2D and 1D stratifications, mostly as online material. We discuss in detail the case of Fe for the $T_{\text {eff }}=8500 \mathrm{~K}$ model in the printed version, and compare it with stratifications derived from observed spectra.
\end{abstract}

Key words. diffusion - stars: abundances - stars: chemically peculiar - stars: magnetic field

\section{Introduction}

Main-sequence chemically peculiar stars of type $\mathrm{HgMn}$ and magnetic Ap are known to exhibit abundance inhomogeneities in their external layers. These inhomogeneities are predicted by atomic diffusion theory and have been discussed for instance by Michaud et al. (1976). Alecian \& Stift (2007) and LeBlanc et al. (2009) specifically address the modelling of abundance stratifications in stellar atmospheres. According to the diffusion model, some metals are expected to accumulate and form clouds in the atmospheres of the above-mentioned stars. These clouds may be distributed uniformly over the stellar surface, for instance when there is no magnetic field, or they may form 2-D patches (actually 3-D inhomogeneities if one considers the depth coordinate), according to the magnetic field geometry. This is the classical diffusion model, which explains the general trends of abundances peculiarities but cannot so far predict the detailed abundance patterns observed in individual stars. Modelling of individual stars is difficult because of the extreme sensitivity of the diffusion process to any macroscopic motion of matter such as turbulence, large-scale circulation, and wind (Babel 1992), etc. We can presently surmise that $\mathrm{HgMn}$ and magnetic Ap stars must be relatively quiet stars since their atmospheres seem to be stable enough to allow abundance stratifications to build up and survive for a considerable time. In addition to these rather qualitative/schematic predictions from theoretical models, observational evidence for such abundance inhomogeneities has

* Figures 9-28 are only available in electronic form at http://www . aanda.org accumulated over recent years (see Ryabchikova et al. 2002; Ryabchikova 2005; Shulyak et al. 2009). Zeeman Doppler mapping (Kochukhov et al. 2004) provides simultaneous magnetic and abundance maps for several elements and indicates the richness of physical processes acting in these stars.

In contrast to the case of stellar interiors, the characteristic timescales of atomic diffusion in stellar atmospheres are relatively short (Alecian 2008). By short, we mean of the order of a human lifespan or the interval between spectroscopic observations (ranging from several years to about a century). This implies that abundance changes with time caused by atomic diffusion in atmospheres can possibly be detected (assuming that abundances peculiarities have not yet reached a stationary stage). The diffusion timescale is roughly proportional to the matter density, provided that the horizontal component of the magnetic field is negligible. Indeed, the diffusion coefficients of ions are inversely proportional to the proton density (diffusion velocities are large in a low density medium), except in horizontal magnetic fields (see Vauclair et al. 1979). Diffusion timescales are short in the upper atmosphere, but they increase as the horizontal component of the magnetic field vector increases (for instance when one goes from a magnetic pole to the magnetic equator). As a consequence - considering short timescales - the build-up of abundance stratifications first proceeds in the upper layers of $\mathrm{HgMn}$ star atmospheres and in the upper layers around the magnetic poles of magnetic Ap stars, and later propagates to deeper layers. Such a time-dependent process has not yet been modelled because it would need enormous computing resources. We can currently compute only equilibrium solutions, which requires assuming that the time-dependent process reaches equilibrium. 
This seems reasonable in some cases, but equilibrium is certainly not always achieved for all elements. However, the equilibrium approximation (see the detailed discussion in Alecian \& Stift 2007) provides a useful starting point for this kind of studies.

In this paper, we present extensive calculations of abundance stratifications (equilibrium solutions in LTE) for 4 effective temperatures and 16 metals: $\mathrm{Mg}, \mathrm{Al}, \mathrm{Si}, \mathrm{P}, \mathrm{Ca}, \mathrm{Ti}, \mathrm{V}, \mathrm{Cr}, \mathrm{Mn}, \mathrm{Fe}, \mathrm{Co}$, $\mathrm{Ni}, \mathrm{Cu}, \mathrm{Zn}, \mathrm{Ga}$, and $\mathrm{Hg}$. We deduce the bi-dimensional stratification of these elements in the atmospheres of main-sequence stars (with $T_{\text {eff }}$ going from $8500 \mathrm{~K}$ to $14000 \mathrm{~K}, \log g=4.00$ ) assuming a dipolar geometry of the magnetic field $\left(2 \times 10^{4} \mathrm{G}\right.$ at the pole). Complete results are given as online material, but here we discuss in detail the case of Fe. In Sect. 2, we recall the theoretical context of our modelling, in Sect. 3, we describe the numerical method we use to obtain the stratifications, and how we derive the 2D representations from the grid of $1 \mathrm{D}$ data we have built. Finally, in Sect. 4, we discuss our results.

\section{Element stratifications caused by the diffusion process}

The first studies of atomic diffusion in CP stars (just after the pioneering paper of Michaud 1970) generally considered only one of the two driving terms in the expression for the diffusion velocity $\left(V_{\mathrm{D}}\right)$, i.e., the radiative acceleration $g_{\text {rad }}$ (the other one being the gravity $g$ ) - see Eq. (4) of Alecian \& Stift (2006). This led to the so-called zeroth order models in which elements that are strongly pushed up by the radiation field were considered to be good candidates to exhibit overabundances in stellar external layers. Overabundances (or under-abundances) in external layers are the consequence of selective migration of elements, which is a non-local, time-dependent, non-linear process (due to the non-linear dependence of radiative accelerations on the element concentrations).

\subsection{Time-dependent process versus equilibrium solution}

Time-dependent diffusion and equilibrium solutions were discussed in Alecian \& Stift (2007); we recall here only the main aspects. To establish how abundances evolve with time and what their values are when the star is observed, one has to solve the respective continuity equations for the number densities of each element. This is performed for stellar interiors (see for instance, Turcotte et al. 1998). Since stellar atmospheres are optically thin, the calculation of radiative accelerations however requires detailed non-local radiative transfer solutions for each wavelength and time step. This would involve expensive numerical computations that for most elements are presently out of reach (note, however, that we have started these calculations with some approximations, and first results will be presented in a forthcoming paper). A reasonable alternative is to search for equilibrium solutions corresponding to $g_{\mathrm{rad}}=g$ (or $\left.V_{\mathrm{D}} \approx 0\right)$. There are two important assumptions underlying the equilibrium solution: (i) stable equilibrium is achieved at the end of the timedependent process, and (ii) the end of this process occurs before the date of observation. Neither of these assumptions can presently be verified, although the first will probably not be met in many cases. It also seems likely that the time-dependent stratification build-up converges in some cases towards a stationary solution with a constant flux of particles throughout the atmosphere (with a non-zero diffusion velocity). This solution may differ substantially from the equilibrium solution and would be strongly dependent on the boundary conditions.
We emphasize that equilibrium solutions discussed hereafter are not solutions of the continuity equation and thus there is no conservation of particle numbers: one simply looks for the stratification that yields $g_{\mathrm{rad}}=g$ throughout the atmosphere. However, for some elements and stellar parameters, real systems may conceivably develop to such equilibrium solutions; in any case, they provide a useful reference framework for future studies.

\subsection{Plane-parallel modelling of Ap star atmospheres}

Spherical symmetry is particularly well justified for HgMn stars, for which observational evidence has convincingly established that magnetic fields are negligible and rotation rates low. Spherical symmetry however does not hold everywhere in magnetic Ap stars: these stars exhibit multipolar large-scale magnetic fields where the main axes of the dipolar (and quadrupolar) components do not coincide with the rotation axis (Borra et al. 1982). Unsurprisingly, this constitutes a source of uncertainties in spectroscopic diagnostics (Stift \& Alecian 2009). Nevertheless, Ap stars usually are slow rotators (the effects of rotation on the stellar structure are therefore small) and the magnetic field geometry is frequently dominated by the dipolar component. One can thus in a first approximation consider that, as far as atomic diffusion (which depends mainly on the magnetic field inclination) is concerned, the assumption of axisymmetry about the magnetic axis is well justified. Keeping in mind that the diffusion process essentially acts along the vertical axis (see the discussion about horizontal diffusion in Alecian \& Stift 2006), the entire surface of an Ap star can be reasonably approximated as the juxtaposition of independent facets computed along a quadrant of the magnetic meridian, using plane-parallel atmospheres.

\section{Numerics}

\subsection{Computation of the models grid}

We computed a grid of plane-parallel models using our code CaratStrat presented and discussed in Alecian \& Stift (2007). This code calculates the diffusion velocities (with and without magnetic fields) considering the detailed line opacities of stratified elements, taking into account Zeeman splitting, and using an accurate solver of the equation of polarized radiative transfer. The redistribution of momentum, among ions of a given species, is computed following the prescription of Alecian \& Stift (2006) and their Eq. (18) for the total radiative acceleration. Still, CaratStrat is subject to a certain number of limitations. In particular, radiative accelerations are computed by assuming LTE, and the input model atmospheres are taken from ATLAS9 or ATLAS12 (Kurucz 1993, 2005) with homogeneous solar abundances assumed. The LTE approach potentially introduces large uncertainties in radiative accelerations for layers with $\log \tau \lesssim-4.0$, the use of homogeneous models being somewhat inconsistent with the equilibrium solutions. We note also that the stratifications of metals can lead to significant modifications of the structure of the atmosphere, especially in deep layers (LeBlanc et al. 2009). These points are discussed in detail later, but let us state here that we consider the limitation of LTE to be the most important (see Borsenberger et al. 1981; Leckrone et al. 1999; Proffitt et al. 1999).

Computations (84 models) were performed for the following sets of parameters: temperature $T_{\text {eff }}=8500,10000,12000$, and $14000 \mathrm{~K}$, field strength $0,1000,5000,10000,15000$, 
and $20000 \mathrm{G}$, and field angle (with respect to the vertical) 0 , 60,75 , and 90 degrees. Equilibrium solutions were obtained after about 20 iterations for each model. In that way, we created, for each effective temperature, a 2D-grid of 21 models (field strength, field angle).

\subsection{From the grid of models to $2 D$-stratifications}

When we consider a simple (centred) dipolar geometry and a spherical star, the field strength and its orientation over the stellar surface can be computed in a straightforward way. In the following, we assume that field strength and orientation do not depend on the depth in the atmosphere.

The maximum field strength is found at the magnetic pole $\left(0^{\circ}\right.$ angle between surface normal and field vector) and the minimum strength (half the maximum) at the magnetic equator $\left(90^{\circ}\right)$. In contrast to the variation in the field strength however, the effect of this dipolar field on the diffusion coefficient for vertical diffusion is null at the pole and reaches a maximum at the magnetic equator.

We compute the equilibrium stratification of a given element at a given position along the magnetic meridian by first determining the field angle and the field strength at that position, then using the grid of equilibrium solutions to interpolate the stratified abundances. The parameters of the grid that we have established allow us to consider any star for which the polar field strength does not exceed $20 \mathrm{kG}$. For this paper, we decided to present 2D stratifications for this particular maximum polar field strength. Our results are presented in the online figures (Figs. 9 to 24), which display gray-scale pictures of the concentration with respect to $\mathrm{H}$ (given by $\left.\log \left(N_{\mathrm{X}} / N_{\mathrm{H}}\right)+12\right)$. In the figures, an identical grey level is used to represent the solar concentration, irrespective of the element: darker shades correspond to overabundances, brighter shades to under-abundances. Because of technical constraints in the preparation of these figures, the depth in the atmosphere ( $y$-axis) is given by the number of the layer rather than the optical depth. These numbers are more or less proportional to $\log \tau_{5000}$, their precise relation to $\log \tau_{5000}$ being indicated in Fig. 1.

Our iterative method converges in general correctly toward the equilibrium solution. However, when the magnetic field is strictly horizontal, the iteration frequently does not converge well above $\log \tau_{5000} \approx-4$, corresponding approximately to layer 30. The diffusion of ions happens to be close to being blocked, and then, the main contribution to the total diffusion velocity comes from the neutral state of the element. Lines originating from neutral states are generally only weakly saturated or not saturated at all. In this case, the radiative acceleration is quite insensitive to the element concentration, and the iteration procedure fails to converge within a reasonable number of steps. Note that, as previously mentioned, the assumption of LTE in our calculations renders the results anyway unreliable in such high layers.

Except for the above-mentioned cases, radiative accelerations are almost equal to gravity (i.e. $10^{4}$ in our models) - and fluxes nearly zero - for the stratifications that we present, in accordance with the definition of the equilibrium stratification. Deviations from these ideal values are caused by the adopted simple convergence criterion. In view of the considerations outlined above, we decided not to present stratifications above $\log \tau_{5000} \approx-4$ in the magnetic case. For HgMn stars or Ap stars with weak magnetic fields ( $\lesssim 1 \mathrm{kG}$ ), the bi-dimensionality can be neglected and the usual 1D stratification representation is sufficient. For that reason, we also present, in a succinct way

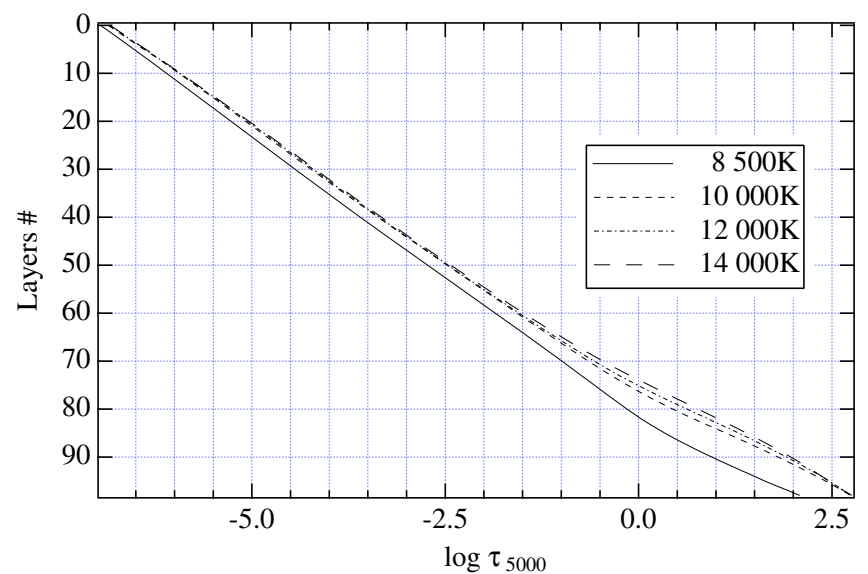

Fig. 1. Layer number versus logarithm of the optical depth at $5000 \mathrm{~A}$ for the four atmospheric models used in the computations.

(Figs. 25 to 28), the zero field cases for the four models. The case of Fe will be discussed in more detail in subsequent sections, so a few of the results are also displayed in the printed version of this paper.

\section{The case of iron in a $8500 \mathrm{~K}$ star}

In this section, we discuss in detail the case of iron for the $T_{\text {eff }}=8500 \mathrm{~K}$ model, which is the coolest of our set of models. This case may be compared more easily to existing observations than hotter models, since at present most empirical stratifications have been derived for cool Ap stars (see Sect. 5). We do not discuss results for layers higher than $\log \tau_{5000}<-4$ because our calculations are in LTE.

The total radiative acceleration - see Eq. (13) of Alecian \& Stift (2006) - of Fe for uniform solar abundance is presented in Fig. 2 for $0 \mathrm{G}$ and $20 \mathrm{kG}$ (vertical field lines), respectively. With vertical field lines, the discrepancy between the 2 curves is caused solely by Zeeman amplification (here, by 0.18 dex, i.e. a factor of 1.5 enhancement around $\log \tau_{5000}=-2$ ). As is obvious from Fig. 2, the radiative acceleration (smaller than gravity) cannot support a solar abundance of Fe above about $\log \tau_{5000}=$ -0.4 , mainly due to the strong saturation of the Fe II lines. Saturation decreases in deeper layers because Fe II ionises (see Fig. 3). Desaturation of the Fe II lines and the contribution of Fe III leads to an increase in the radiative acceleration, which reaches a maximum at $\log \tau_{5000} \approx 0.65$ where a Fe solar abundance is well supported. Deeper down, the radiative acceleration again decreases because the fraction of Fe II becomes too small to contribute significantly to the total acceleration of Fe.

Following the behaviour of the total radiative acceleration shown in Fig. 2, the diffusion flux of iron (i.e. the net flux of particles across a horizontal surface unit), which has the same sign as the diffusion velocity, must be negative above $\log \tau_{5000} \approx$ -0.4 , and positive below. This is, indeed, what one finds in Fig. 4 for $0 \mathrm{G}$ and $20 \mathrm{kG}$ (vertical field lines, magnetic pole). However, the flux is positive above $\log \tau_{5000}=-1.5$ for $10 \mathrm{kG}$ when field lines are horizontal as at the magnetic equator of a dipole with $20 \mathrm{kG}$ at the pole. This is a well known effect of horizontal magnetic fields (for a detailed discussion see Alecian \& Stift 2006).

By considering the behaviour of the diffusion flux as a function of field angle displayed in Fig. 4, one deduces that iron should accumulate around $\log \tau_{5000}=0.65$ and be depleted above $\log \tau_{5000} \approx-0.4$ for both the zero field case and a vertical $\left(0^{\circ}\right) 20 \mathrm{kG}$ field. One can also surmise that, according to the 


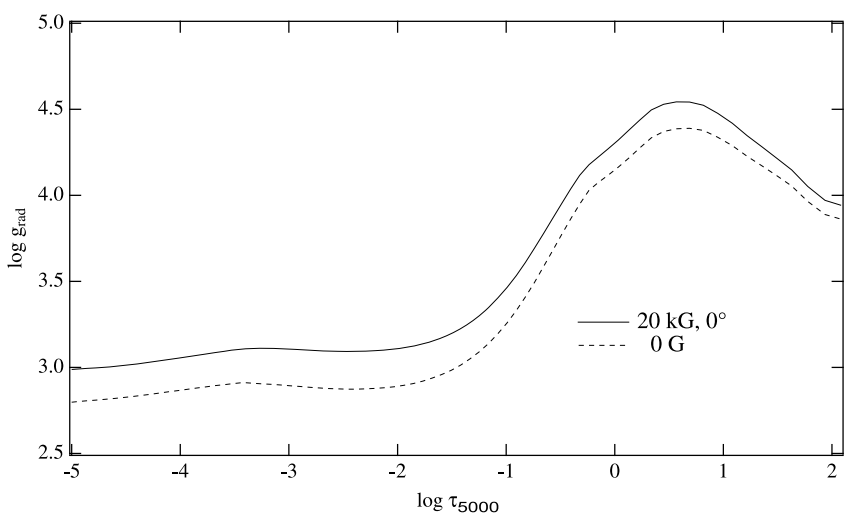

Fig. 2. Radiative acceleration of Fe for homogeneous solar abundance $\left(T_{\text {eff }}=8500 \mathrm{~K}\right)$ vs. the optical depth (both quantities are given logarithmically). The dashed curve corresponds to zero magnetic field, the solid one to $20 \mathrm{kG}$ (vertical field lines). The differences between the curves are due exclusively to Zeeman amplification.

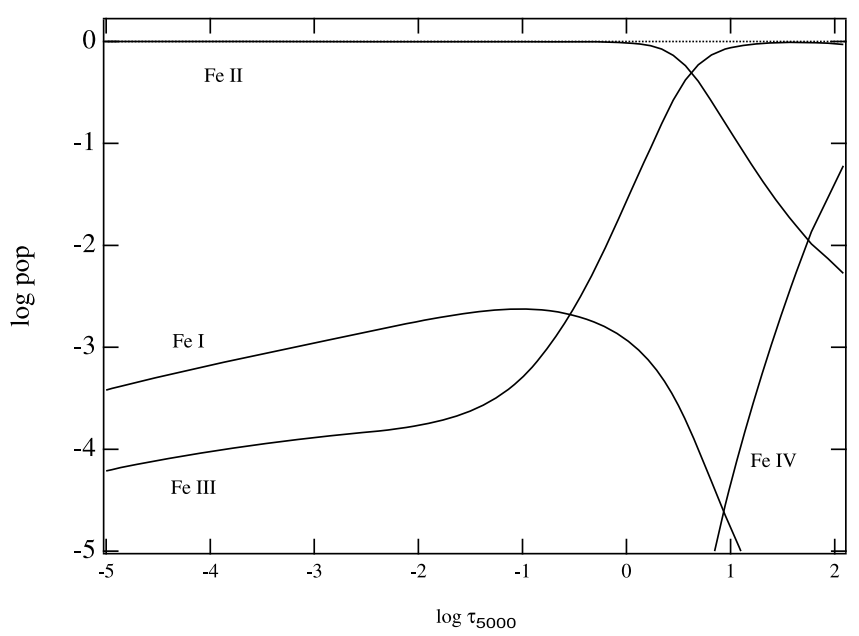

Fig. 3. Population of the first 4 ionisation stages of $\mathrm{Fe}\left(T_{\mathrm{eff}}=8500 \mathrm{~K}\right.$, LTE assumed) vs. the logarithm of optical depth.

accelerations of Fig. 2, the accumulation around $\log \tau_{5000}=0.65$ will be by a factor between 3.4 and 10 with respect to the solar value, since the medium is almost optically thick in the vicinity of these layers. It is indeed known that for saturated lines in an optically thick medium, the radiative acceleration varies as $N^{\alpha}$, with $-1 \lesssim \alpha \lesssim-0.5$ according to the Voigt parameter of the line profile (see Alecian \& LeBlanc 2000). It is not possible to quantitatively estimate the equilibrium abundance in an optically thin medium without resorting to numerical computation (see below), but one expects $\mathrm{Fe}$ to accumulate above $\log \tau_{5000} \approx-1.5$ for $10 \mathrm{kG}\left(90^{\circ}\right)$. This is again verified in Fig. 5, where we present equilibrium stratifications obtained with our CaratStrat code for the three cases of Fig. 4.

From the whole grid of models, and as discussed in Sect. 3.2, we build the $2 \mathrm{D}$ stratification of Fe for a dipolar field $(20 \mathrm{kG}$ at the pole). The result is presented in Fig. $6-$ this figure shows the same stratification as presented in the online Fig. 11 (topright panel). Note that the curves for 20 and $10 \mathrm{kG}$ of Fig. 5 correspond to a cut in Fig. 6 at $0^{\circ}$ and $90^{\circ}$, respectively.

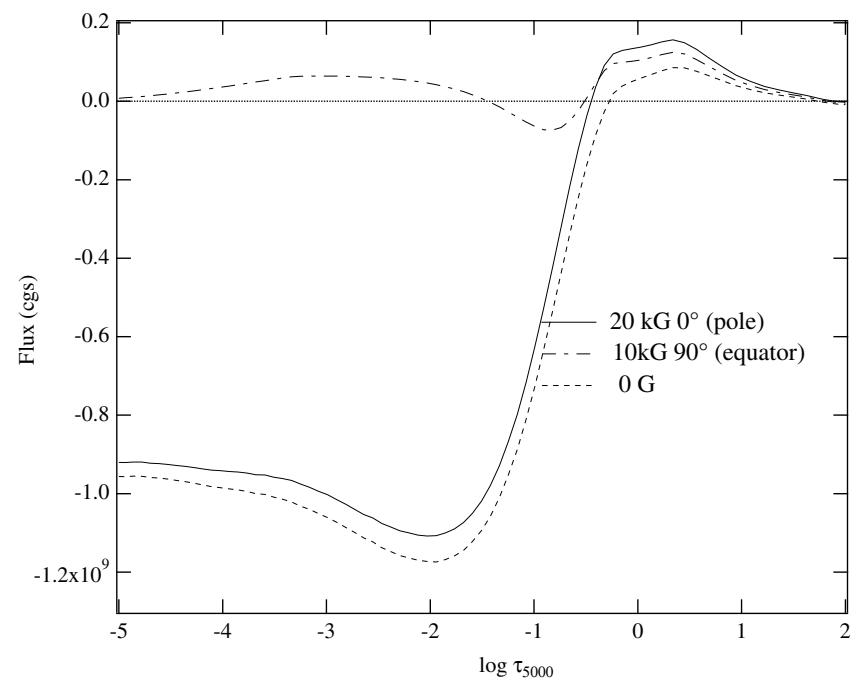

Fig. 4. Diffusion flux of Fe for homogeneous solar abundance $\left(T_{\mathrm{eff}}=\right.$ $8500 \mathrm{~K}$ ) vs. the logarithm of optical depth. The solid line is for a vertical $20 \mathrm{kG}$ field, the dashed-point line for a $10 \mathrm{kG}$ horizontal field; the zero field case is given by the dashed line.

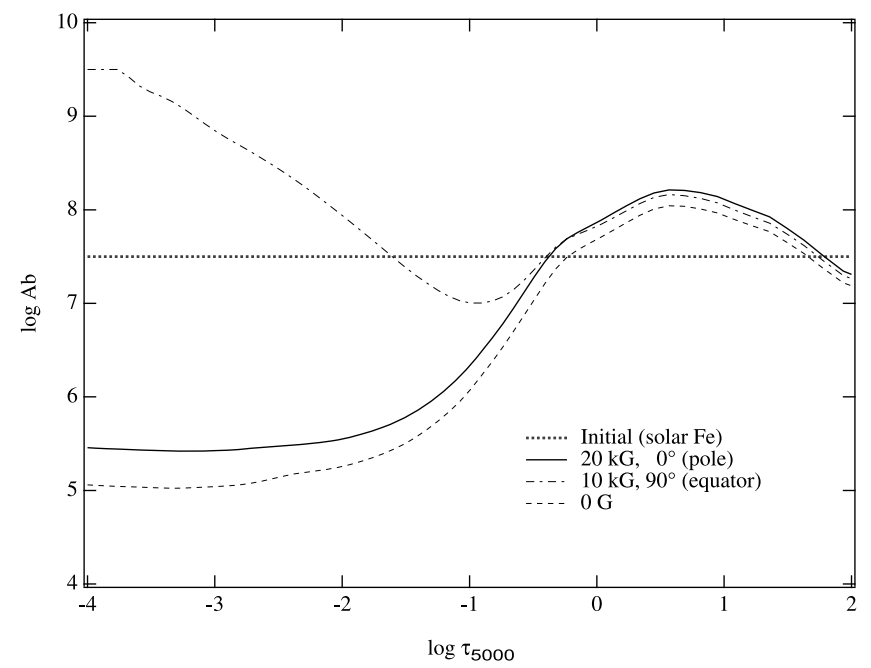

Fig. 5. Equilibrium stratifications of $\mathrm{Fe}\left(T_{\text {eff }}=8500 \mathrm{~K}\right)$ vs. the logarithm of optical depth. Abundances are in $\left.\log \left(N_{X} / N_{H}\right)+12\right)$. The meaning of the lines is the same as in Fig. 4, the horizontal dotted line corresponds to the solar abundance of $\mathrm{Fe}$ (the starting value of the iterations).

\section{Discussion}

\subsection{Comparison with observations for the case of Fe}

In Fig. 7 we have gathered some "observed" stratifications of Fe compiled by Ryabchikova (2008). These abundance profiles were obtained by fitting observed iron lines to synthetic spectra using essentially two techniques: the step function approximation (Ryabchikova et al. 2005) and the vertical inverse problem (Kochukhov et al. 2006). Even though these "observed" stratifications are only approximate (note that no error bars have ever been published) and do not take into account the structure of the magnetic field (see the discussion in Stift \& Alecian 2009), they provide an opportunity to confront the theoretical predictions with inversion results. These stratifications, in general, tend to show an overabundance of Fe in layers deeper than $\log \tau_{5000} \approx-0.5$. The iron abundance is lower by at least $1 \mathrm{dex}$ in the layers above, the transition being rather sharp. As far as one can tell from such a small sample of stars, there may be 


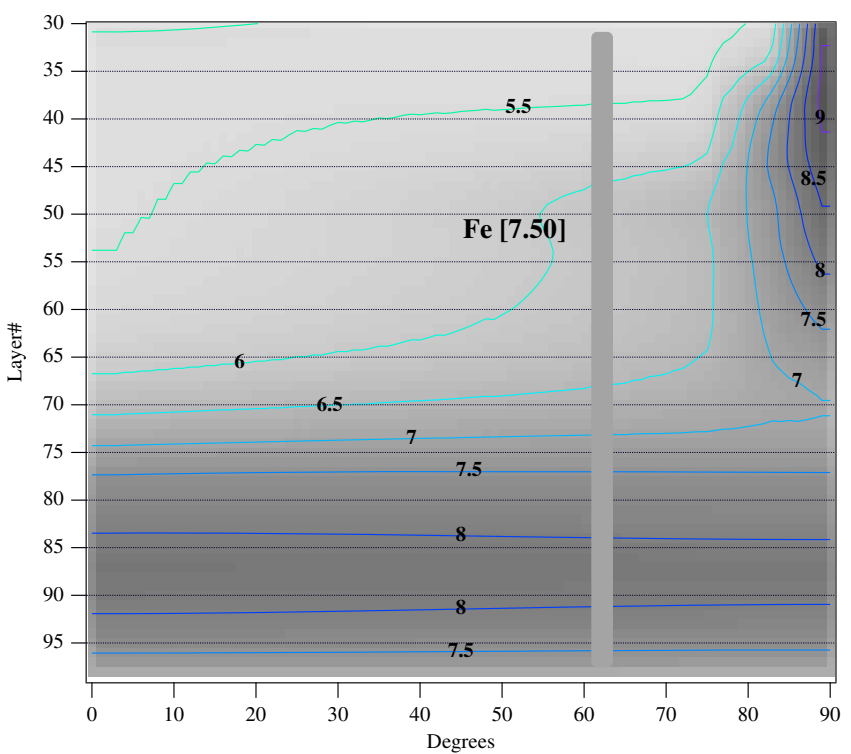

Fig. 6. Bi-dimensional stratification of Fe in a $T_{\text {eff }}=8500 \mathrm{~K}$ model. Abundances (expressed in $\left.\log \left(N_{X} / N_{H}\right)+12\right)$ are shown on a gray-scale: the $y$-axis gives the layer number (the corresponding depth in the atmosphere can be taken from Fig. 1), the bottom axis the position along the magnetic meridian, counted from the magnetic pole $\left(0^{\circ}\right)$ with $20 \mathrm{kG}$ towards the equator $\left(90^{\circ}\right)$. The vertical bar (at about $62^{\circ}$ ) visualises the grey level corresponding to the solar abundance of $\mathrm{Fe}$ (given in brackets beside the name of the element). Note how the vertical bar becomes almost invisible near the bottom of the plot (and around layer 78), where the amount of $\mathrm{Fe}$ is nearly solar. The scaling of the grey levels is such that abundances lower than $10^{-3}$ times the solar value are white, and abundances greater than $10^{4}$ times the solar value are black. The results above layer 30 are not reliable and are therefore not shown (see text).

another possible general trend in that iron abundances appear to be lower for the coolest stars and that the size of the step is smaller for the hottest stars. One cannot notice any correlation between measured quantities and magnetic field strength in these observations.

We do not consider the magnetic case in this discussion, mainly because we do not wish to have to deal with an unnecessary extra parameter, but also because in our opinion the magnetic case should be discussed in a bi-dimensional framework (see Sect. 5.2). To compare the "observed" stratifications with our theoretical equilibrium solutions, we have plotted in Fig. 8 our zero field case results for the four atmospheric models used in the present paper, together with the stratification found for HD $188091\left(T_{\text {eff }}=8800 \mathrm{~K}, \log g=4.0\right)$, which is the star closest to one of our models. The stratification profile for HD 188091 has been offset by -1.3 dex to make it easier to compare the size and position of the step. We also computed a model (ATLAS12) of lower gravity $\left(\log g=3.8, T_{\text {eff }}=8500 \mathrm{~K}\right)$ and have plotted, in the same Fig. 8, the corresponding equilibrium stratification of $\mathrm{Fe}$ (see discussion below). Our theoretical results are similar (for $0 \mathrm{G}$ ) to those obtained by LeBlanc et al. (2009), but one cannot perform a more precise comparison because the adopted atmospheric models differ significantly. One finds that the theoretical overabundance around $\log \tau_{5000} \approx 0.0$ is systematically lower than the "observed" one; however, the position of the jump in HD 188041 and its magnitude are comparable to what is obtained for the $8500 \mathrm{~K}$ model. On average, theoretical overabundances appear to increase with effective temperature and the size of the jump decreases, both of these findings being compatible with the "observed" stratifications.

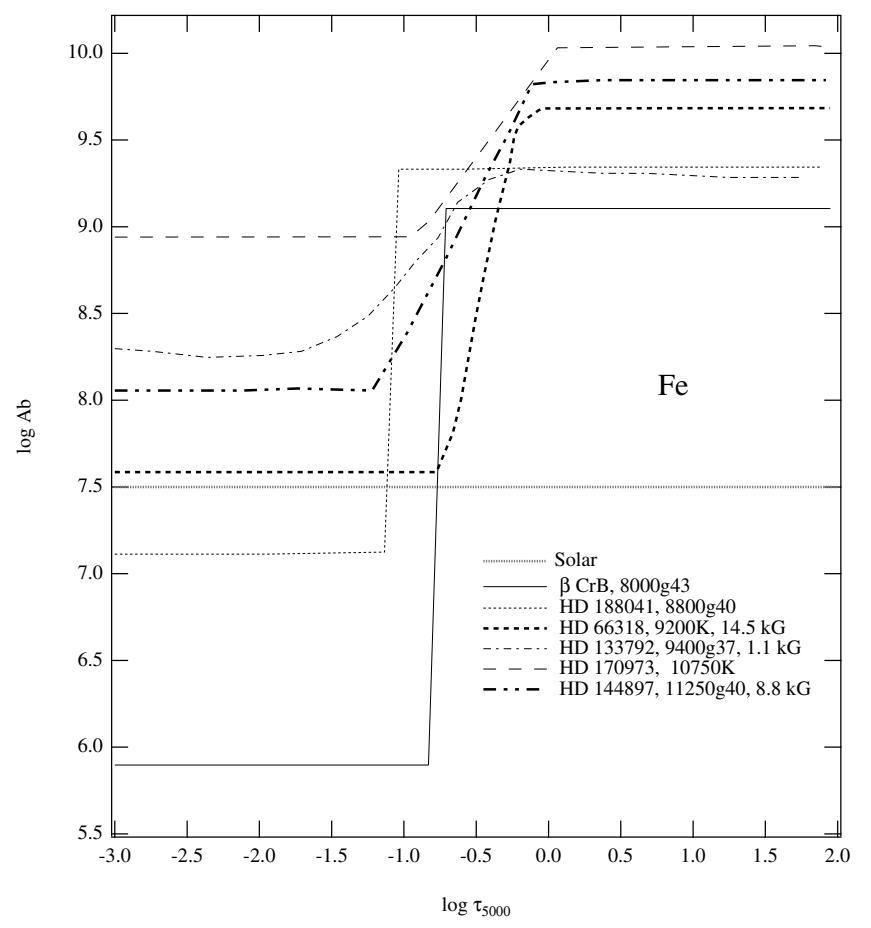

Fig. 7. Compilation of Fe stratifications determined from the spectra of six CP stars (compilation by Ryabchikova 2008).

For several reasons, one cannot expect close similarity between observed and theoretical stratifications. Apart from the uncertainties/flaws inherent in both the observational and theoretical approaches, one has to keep in mind that diffusion in real stars will not necessarily over time result in theoretical equilibrium solutions (see Sect. 2.1). On the other hand, theoretical solutions are sensitive to the gravity of the model. Here, all the models used to prepare our grid have $\log g=4.0$. To check the effect of varying gravity, we computed a model with $\log g=3.8$ and $T_{\text {eff }}=8500 \mathrm{~K}$. The resulting equilibrium stratification of $\mathrm{Fe}$ is shown in Fig. 8 (heavy long-dashed curve). One sees that the supported overabundance is, as expected, higher for the low gravity model, although, apparently not by a sufficient amount. The large "observed" overabundance of Fe around $\log \tau_{5000} \approx 0.0$ remains disturbing since such Fe abundances are not supported by the radiation field in these layers and for the assumed combination of effective temperature and gravity. If in the future, such overabundances were to be confirmed and firmly established, one would have to invoke some process in addition to atomic diffusion (e.g., mass-loss).

\subsection{The significance of bi-dimensional results}

A magnetic field affects the equilibrium stratifications of chemical elements in two ways: (i) the Zeeman amplification of radiative accelerations usually increases the element abundance supported by the radiation field, and (ii) the decrease of the diffusion coefficient of ions impedes the ion's motion across the field lines. The first effect is the most dominant at the magnetic poles and is illustrated in Fig. 2. The second effect is less trivial since its efficiency depends on the inclination of the field lines towards the vertical and on the relative populations of neutral state and ions, respectively. For most elements and atmospheres shown in Figs. 9 to 24, one notes a sharp contrast in the stratifications close to the magnetic equator. The effect of the magnetic field inclination changes rather moderately between $0^{\circ}$ and $75^{\circ}$, but the rate 


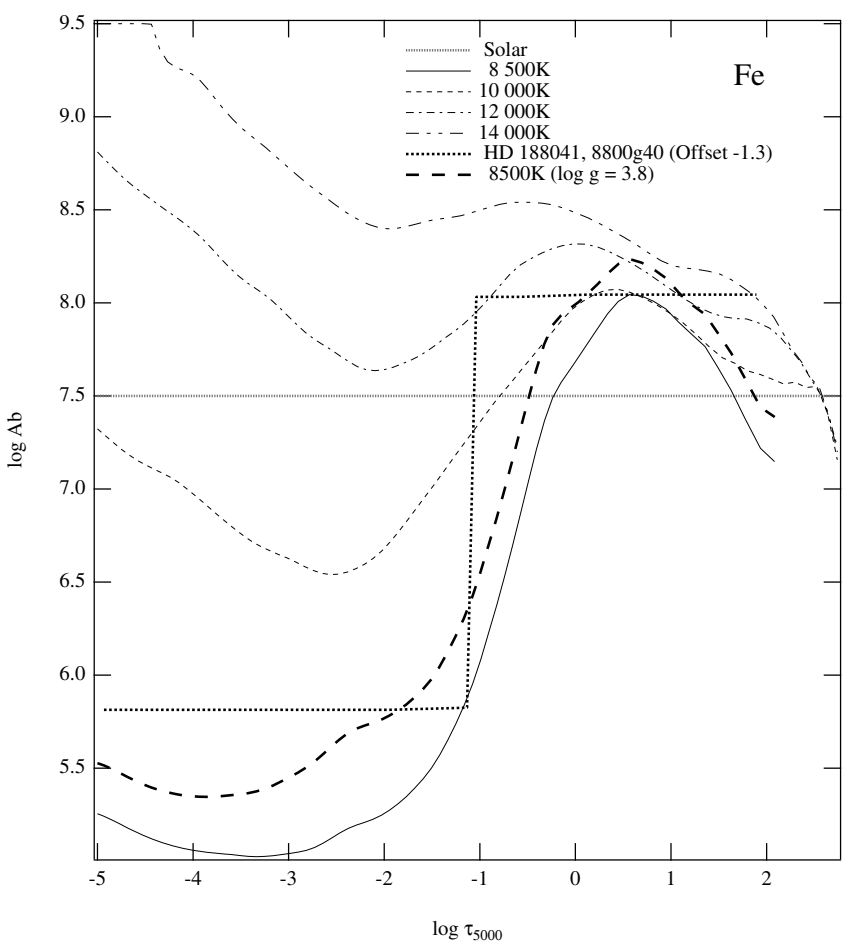

Fig. 8. Zero-field equilibrium stratification of Fe for the 4 model atmospheres used in this work, and the lower gravity model. The stratification proposed for HD 188041 (Ryabchikova 2008) is also shown (same as in Fig. 7), but with an abundance offset of -1.3 dex to make the comparison with the calculated step of the closest model $(8500 \mathrm{~K})$ easier.

of change steepens between $75^{\circ}$ and $90^{\circ}$, often leading to narrow equatorial belts of enhanced metals in atmospheres permeated by a field with dipolar structure. If such equilibrium stratifications were to be achieved in real stars without a dipole field, our calculations would indicate that patches form each time the field lines come close to $90^{\circ}$. Stars could then be quite spotty if the field fluctuates about the horizontal. Such a high sensitiveness to the inclination angle - confined to angles around $90^{\circ}$ - suggests that the effect of the magnetic inclination on the abundances will affect a rather small fraction of the stellar surface.

Let us outline a possible consequence of the sensitivity of abundances to a field angle near $90^{\circ}$. If the field topology of an Ap star is not strictly dipolar and if the field angle does not take values close to $90^{\circ}$ over a sufficiently large distance from the magnetic equator, it is conceivable that the width of the equatorial belt will become too narrow at some longitudes to be detected by the Doppler mapping technique. In that case, one might obtain a sequence of largish spots (where the field angle stays close enough to $90^{\circ}$ ), instead of a belt. This may possibly help in explaining some of the features observed in stars such as 53 Cam (Kochukhov et al. 2004) where, clearly, no equatorial belt can be seen.

In some cases, Zeeman Doppler mapping reveals significant differences between opposite polar regions (see e.g. Lüftinger et al. 2010). In a strictly dipolar configuration such as the one we have considered, the simple diffusion model cannot reproduce such patterns. For these cases one can invoque, for instance, either a non-dipolar configuration (or a decentred dipole which results in significantly different field intensities at the poles), or the existence, in addition to the diffusion process, of wind velocities that differ slightly from one pole to the other. More accurate observational data are needed to better constrain the theoretical models.

In our results, we note that silicon is usually not tightly supported by the radiation field, even at the magnetic equator. This is inconsistent with the high overabundances (by a factor of 10 and sometimes even more) observed in Ap Si stars. We propose that this may be due to an insufficient representation of autoionisation lines and/or numerous weak lines in our atomic database. Because Silicon is a very abundant element, these lines could contribute significantly more to the radiative accelerations than is the case for other elements. Autoionisation lines are presently only taken into account via photoionisation cross-sections provided by the Opacity Project database. The role of silicon autoionisation lines was discussed in detail in Alecian \& Vauclair (1981). These authors found that the autoionisation lines known at the beginning of the 1980's were not expected to contribute much to support this element; this question however deserves to be re-examined with updated atomic autoionisation line lists.

Our discussion so far has been based on equilibrium solutions. One cannot exclude that the strong contrasts found close to the magnetic equator are overestimated by this equilibrium assumption. Since the real stratification build-up is timedependent, inclined magnetic lines increase the diffusion times of ions. For strictly horizontal magnetic lines, diffusion velocities of ions approach zero, i.e. diffusion timescales become very long, and are moderated only by the neutral state of the atom. This makes the equilibrium solution much more difficult to attain. The longer diffusion times also weaken the stratification build-up as they compete with other processes such as wind. Note that the equilibrium solutions are sensitive only to the sign of the diffusion velocity and to the dependence of radiative acceleration on concentration; they are insensitive to diffusion time. It thus remains to be checked whether the large overabundances obtained at equilibrium for horizontal field lines can ever be built up during the evolution of the star.

\section{Conclusions}

Over recent years, much observational evidence of abundance stratifications has been presented and a considerable number of papers has dealt with the detailed mapping of inhomogeneous distributions of chemical elements over the surfaces of CP stars. The quantity of the observations will continue to increase, and the quality of the various inversion methods will of course improve. Theory has to meet the challenge of modelling these empirical abundance inhomogeneities. At present, the diffusion model offers the only viable framework to understand the observed abundance anomalies, at least qualitatively (more quantitative, time-dependent diffusion calculations are progressing step by step). In the present paper, we have addressed the problem by calculating a grid of abundance stratifications based on LTE equilibrium solutions for 16 metals, for stellar atmospheres with 4 different effective temperatures typical of Ap stars, and for magnetic field strengths between 0 and $20 \mathrm{kG}$. By interpolating in this grid, we have established 2D stratifications - abundances as a function of depth and co-latitude along the magnetic meridian - of the metals in question, assuming a simple dipolar field geometry. For stars without magnetic fields (HgMn stars), and considering layers deeper than $\log \tau_{5000} \approx-4.0$, there is no need to go beyond $1 \mathrm{D}$ stratifications.

Improved physics need to be incorporated into these calculations, such as NLTE effects and self-consistent atmospheric structure (taking into account abundance stratifications together with magnetic fields). This will be among the next important 
steps in forthcoming work. The comparison of our results for Fe with stratifications deduced from real spectra is encouraging insofar as the global shapes of the stratification curves are compatible; one should be careful, however, when interpreting these results because of the limitations of the equilibrium approach discussed in the previous sections. Equilibrium solutions may sometimes be reached in real stars but that end-product of radiative diffusion cannot always be expected. Still, they constitute an important reference point for further studies of chemical stratifications.

Acknowledgements. GA acknowledges the financial support of the Programme National de Physique Stellaire (PNPS) of CNRS/INSU, France. M.J.S. acknowledges support by the Austrian Science Fund (FWF), project P16003N05 "Radiation driven diffusion in magnetic stellar atmospheres" and through a Visiting Professorship at the Observatoire de Paris-Meudon and Université Paris Diderot (LUTH). Thanks go to AdaCore for generously providing us with the GNAT Pro Ada95 compiler and toolsuite. This work was partly performed using HPC resources from GENCI- CINES (Grant 2009-c2009045021).

\section{References}

Alecian, G. 2008, CoAst, 158, 34

Alecian, G., \& Vauclair, S. 1981, A\&A, 101, 16

Alecian, G., \& LeBlanc, F. 2000, MNRAS, 319, 677
Alecian, G., \& Stift, M. J. 2006, A\&A, 454, 571

Alecian, G., \& Stift, M. J. 2007, A\&A, 475, 659

Babel, J. 1992, A\&A, 258, 449

Borra, E. F., Landstreet, J. D., \& Mestel, L. 1982, ARA\&A, 20, 191

Borsenberger, J., Michaud, G., \& Praderie, F. 1981, ApJ, 243, 533

Kochukhov, O., Bagnulo, S., Wade, G. A., et al. 2004, A\&A, 414, 613

Kochukhov, O., Tsymbal, V., Ryabchikova, T., Makaganyk, V., \& Bagnulo, S. 2006, A\&A, 460, 831.

Kurucz, R. 1993, Kurucz CDROM No. 13, Smithsonian Astrophys. Obs.

Kurucz, R. 2005, Mem.A.A.It. Suppl., 8, 14

LeBlanc, F., Monin, D., Hui-Bon-Hoa, A., \& Hauschildt, P. H. 2009, A\&A 495, 937

Leckrone, D. S., Proffitt, C. R., Wahlgren, G. M., Johansson, S. G., \& Brage, T. 1999, AJ, 117, 1454

Lüftinger, T., Kochukhov, O., Ryabchikova, T., et al. 2010, A\&A, 509, A71

Michaud, G. 1970, ApJ, 160, 641

Michaud, G., Charland, Y., Vauclair, S., \& Vauclair, G. 1976, ApJ, 210, 447

Proffitt, C. R., Brage, T., Leckrone, D. S., et al. 1999, ApJ, 512, 942

Ryabchikova, T. 2005, EAS Publ. Ser., 17, 253

Ryabchikova, T. 2008, in Proceedings of the CP\#Ap Workshop, Vienna 2007, CoSka, 38, 257

Ryabchikova, T., Piskunov, N., Kochukhov, O., et al. 2002, A\&A, 384, 545

Ryabchikova, T., Leone, F., \& Kochukhov, O. 2005, A\&A, 439, 973

Shulyak, D., Ryabchikova, T., Mashonkina, L., \& Kochukhov, O. 2009, A\&A, 499, 879

Stift, M. J., \& Alecian, G. 2009, MNRAS, 394, 1503

Turcotte, S., Richer, J., \& Michaud, G. 1998, ApJ, 504, 559

Vauclair, S., Hardorp, J., \& Peterson, D. M. 1979, ApJ, 227, 526 

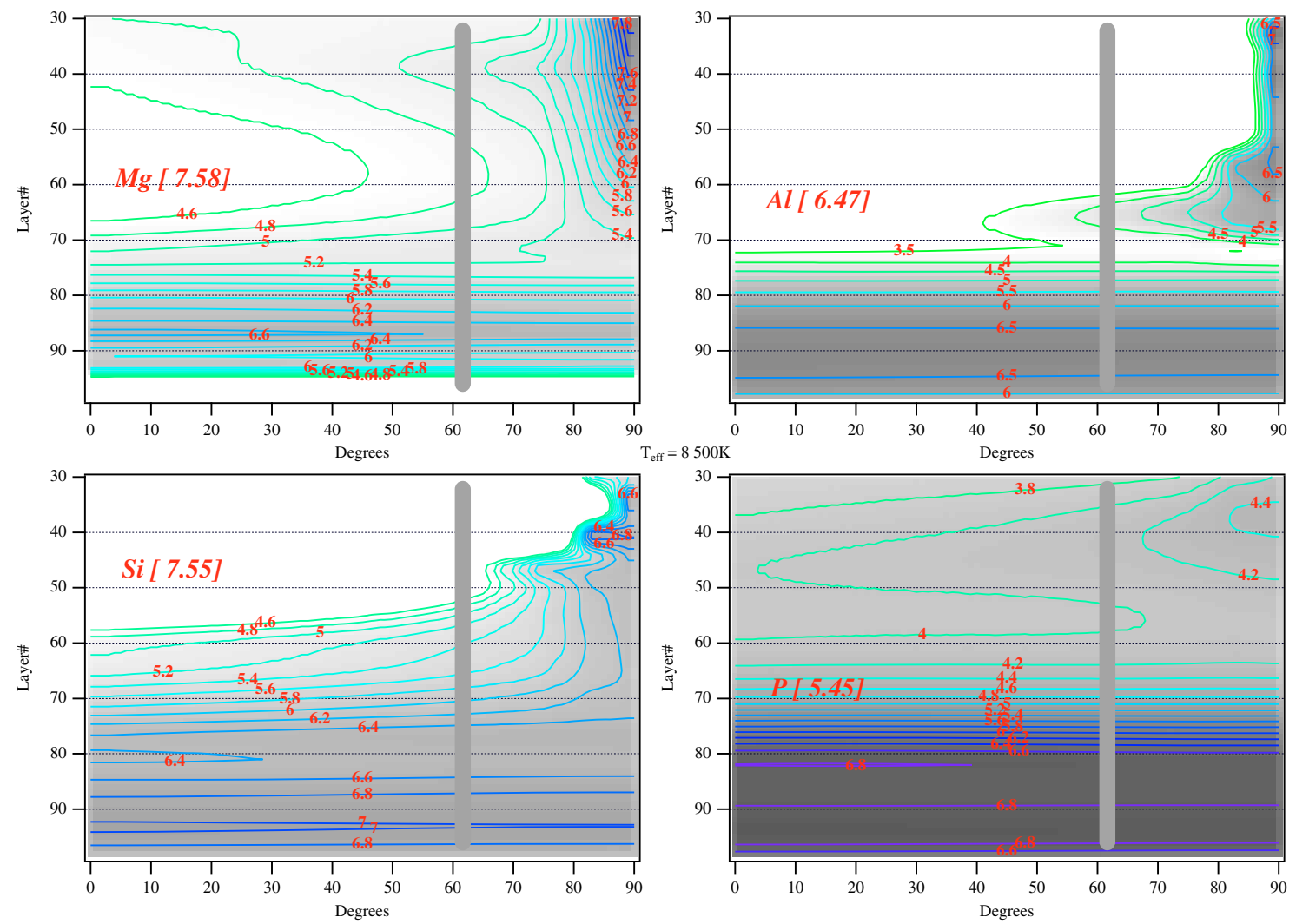

Fig. 9. Bi-dimensional stratification of $\mathrm{Mg}, \mathrm{Al}, \mathrm{Si}$, and $\mathrm{P}$ for a $T_{\text {eff }}=8500 \mathrm{~K}$ model. Abundances (expressed in $\left.\log \left(N_{X} / N_{H}\right)+12\right)$ are shown on a gray-scale: the $y$-axis indicates the layer number (the corresponding depth in the atmosphere can be taken from Fig. 1, the bottom axis the position along the magnetic meridian, counted from the magnetic pole $\left(0^{\circ}\right)$ with $20 \mathrm{kG}$ towards the equator $\left(90^{\circ}\right)$. The vertical bar (at about $\left.62^{\circ}\right)$ visualises the grey level corresponding to the solar abundance of $\mathrm{Fe}$ (given in brackets beside the name of the element). The scaling of the grey levels is such that abundances lower than $10^{-3}$ time the solar value are white, and abundances greater than $10^{4}$ time the solar value are black.
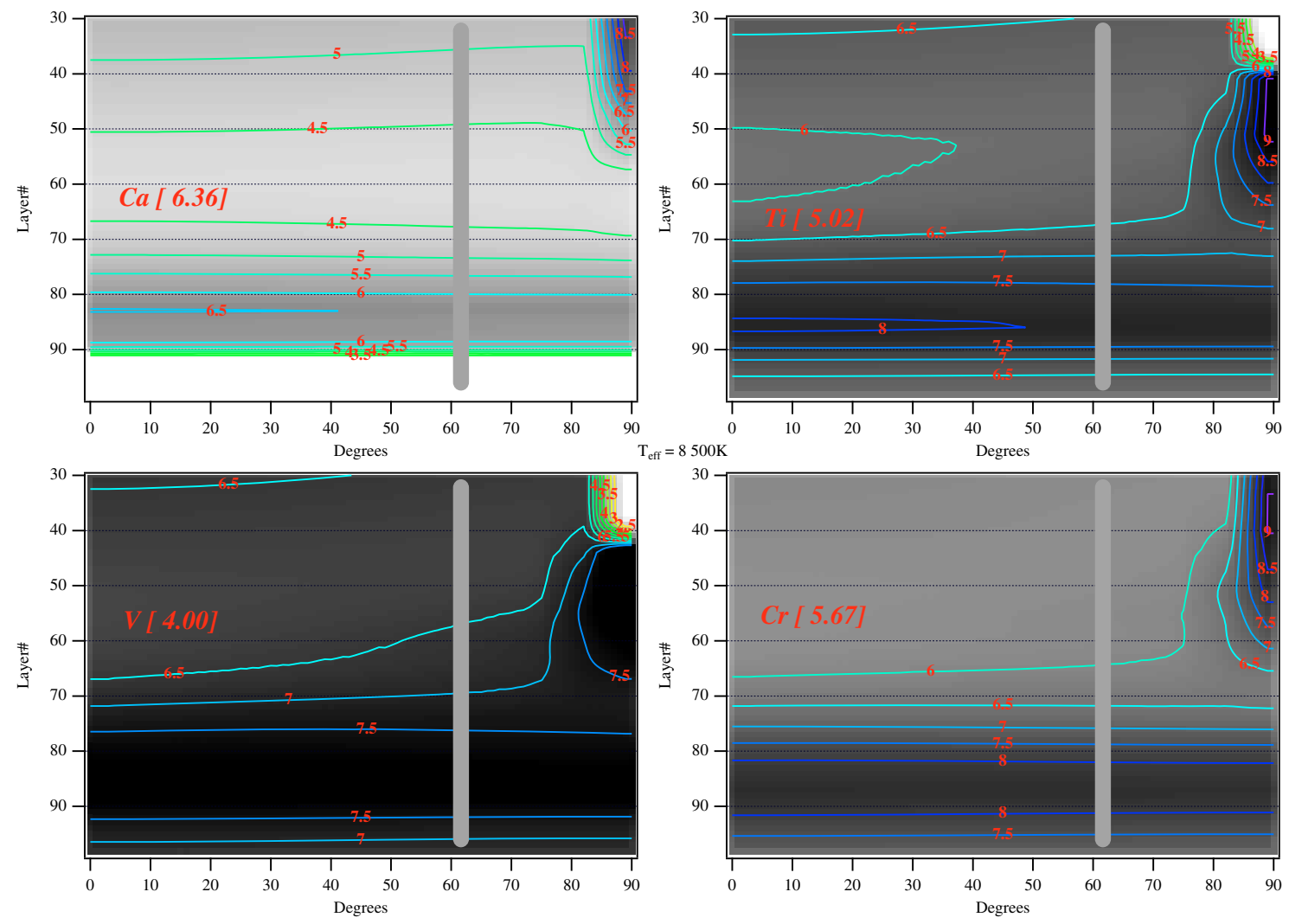

Fig. 10. Bi-dimensional stratification of $\mathrm{Ca}, \mathrm{Ti}, \mathrm{V}$, and $\mathrm{Cr}$ for a $T_{\mathrm{eff}}=8500 \mathrm{~K}$ model. Same annotations as in Fig. 9. 
G. Alecian and M. J. Stift: Bi-dimensional stratifications in Ap stars
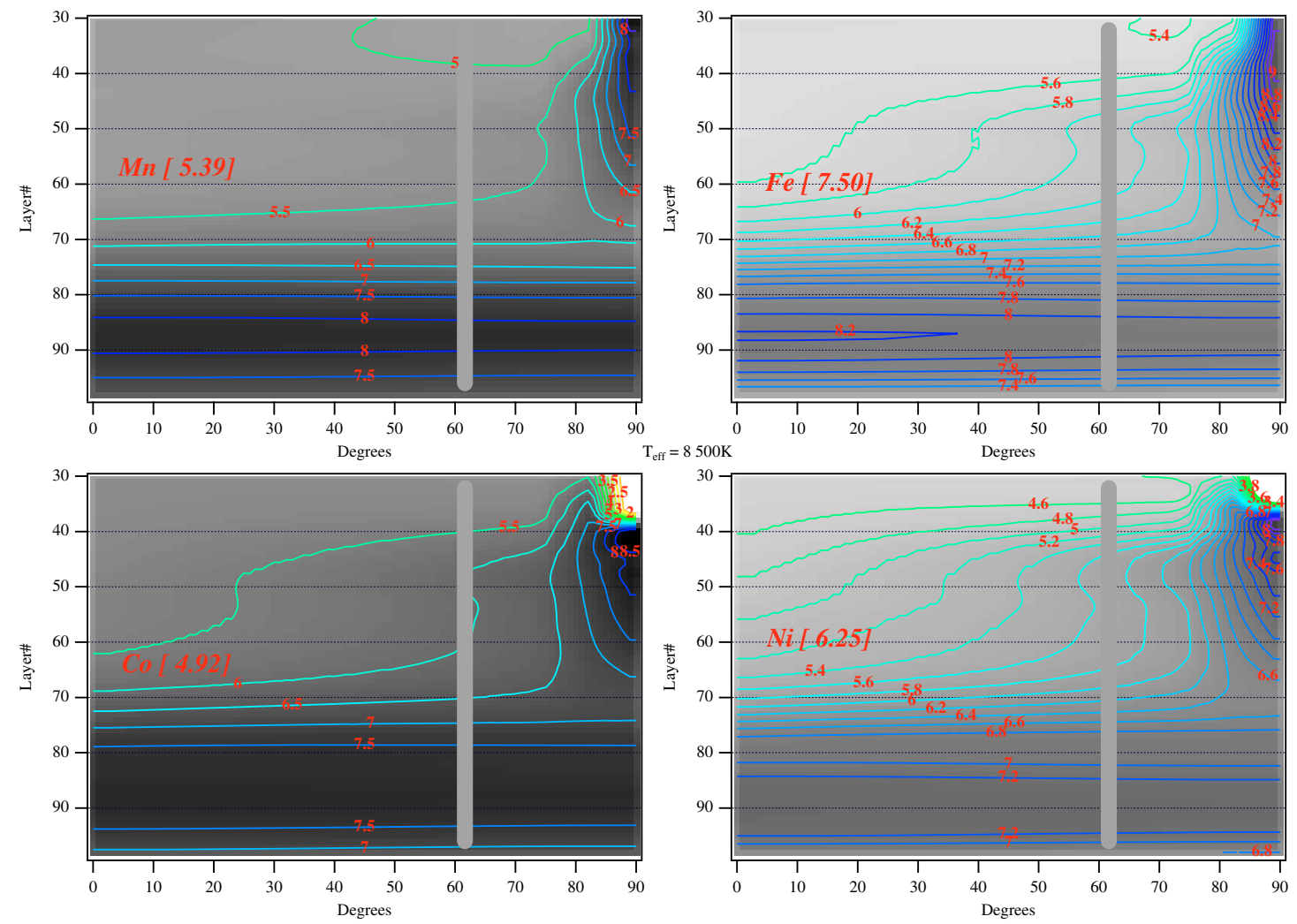

Fig. 11. Bi-dimensional stratification of $\mathrm{Mn}, \mathrm{Fe}, \mathrm{Co}$, and $\mathrm{Ni}$ for a $T_{\mathrm{eff}}=8500 \mathrm{~K}$ model. Same annotations as in Fig. 9.
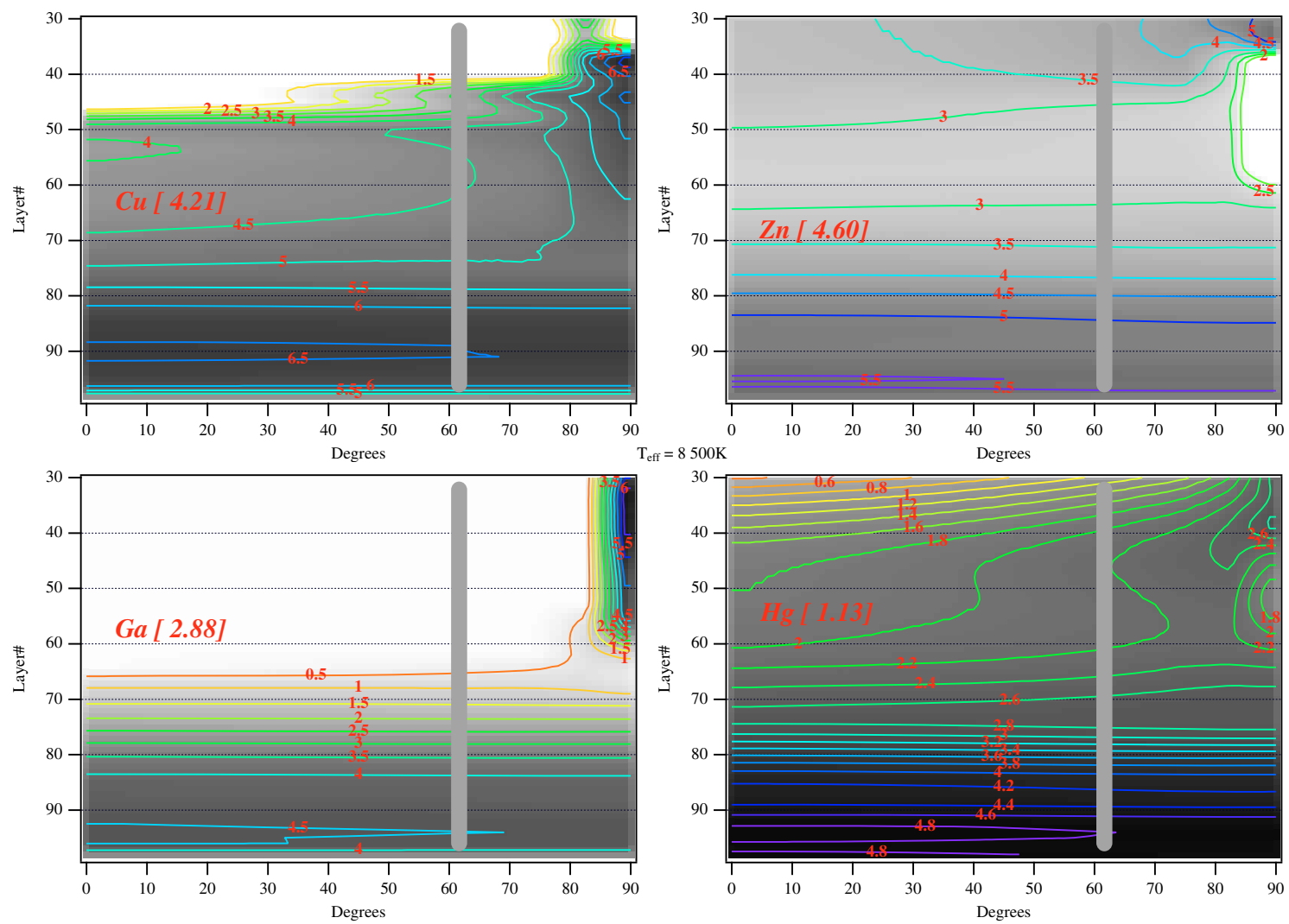

Fig. 12. Bi-dimensional stratification of $\mathrm{Cu}, \mathrm{Zn}, \mathrm{Ga}$, and $\mathrm{Hg}$ for a $T_{\text {eff }}=8500 \mathrm{~K}$ model. Same annotations as in Fig. 9 . 
A\&A 516, A53 (2010)
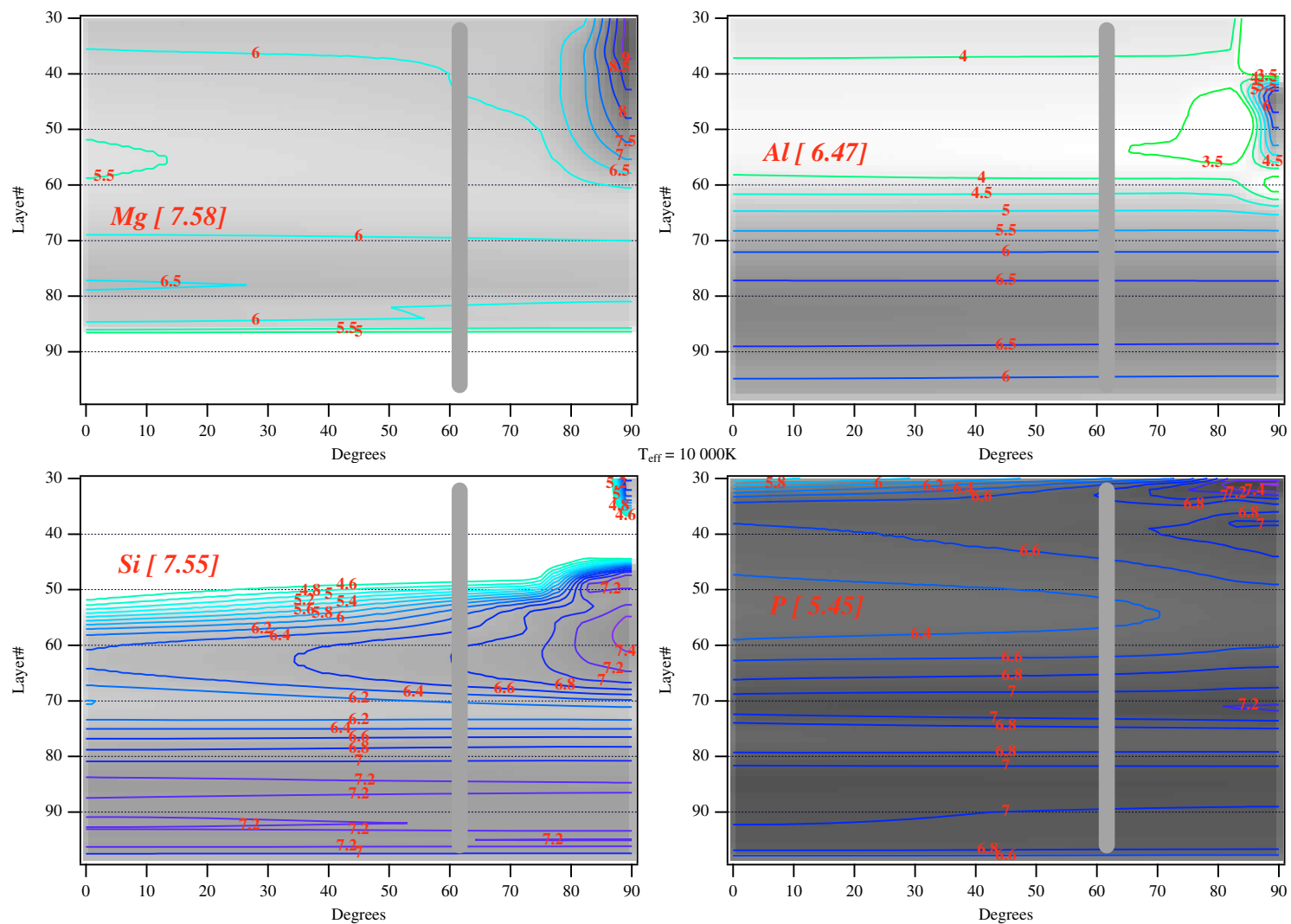

Fig. 13. Bi-dimensional stratification of $\mathrm{Mg}, \mathrm{Al}, \mathrm{Si}$, and $\mathrm{P}$ for a $T_{\text {eff }}=10000 \mathrm{~K}$ model. Same annotations as in Fig. 9.
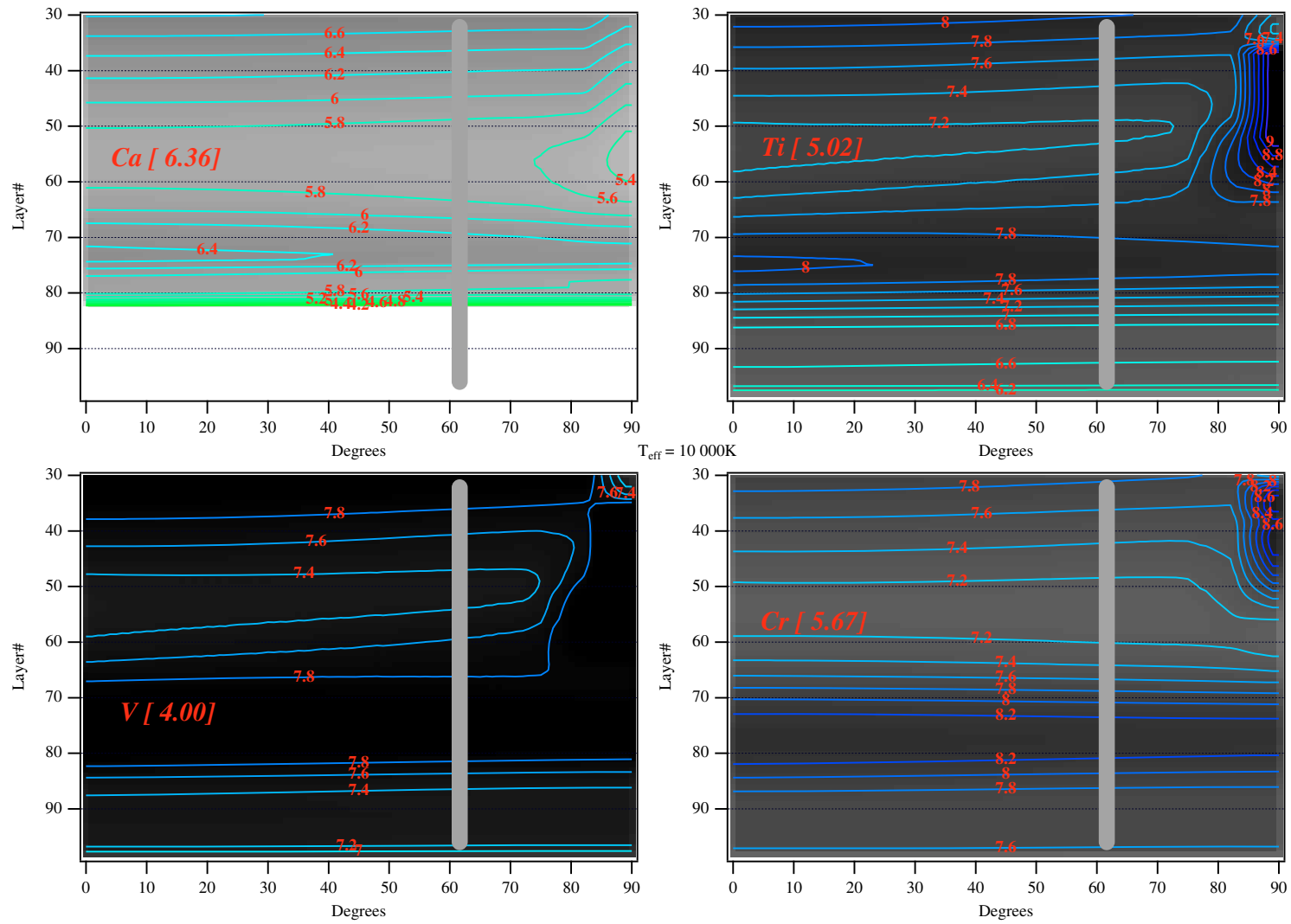

Fig. 14. Bi-dimensional stratification of $\mathrm{Ca}, \mathrm{Ti}, \mathrm{V}$, and $\mathrm{Cr}$ for a $T_{\text {eff }}=10000 \mathrm{~K}$ model. Same annotations as in Fig. 9. 
G. Alecian and M. J. Stift: Bi-dimensional stratifications in Ap stars
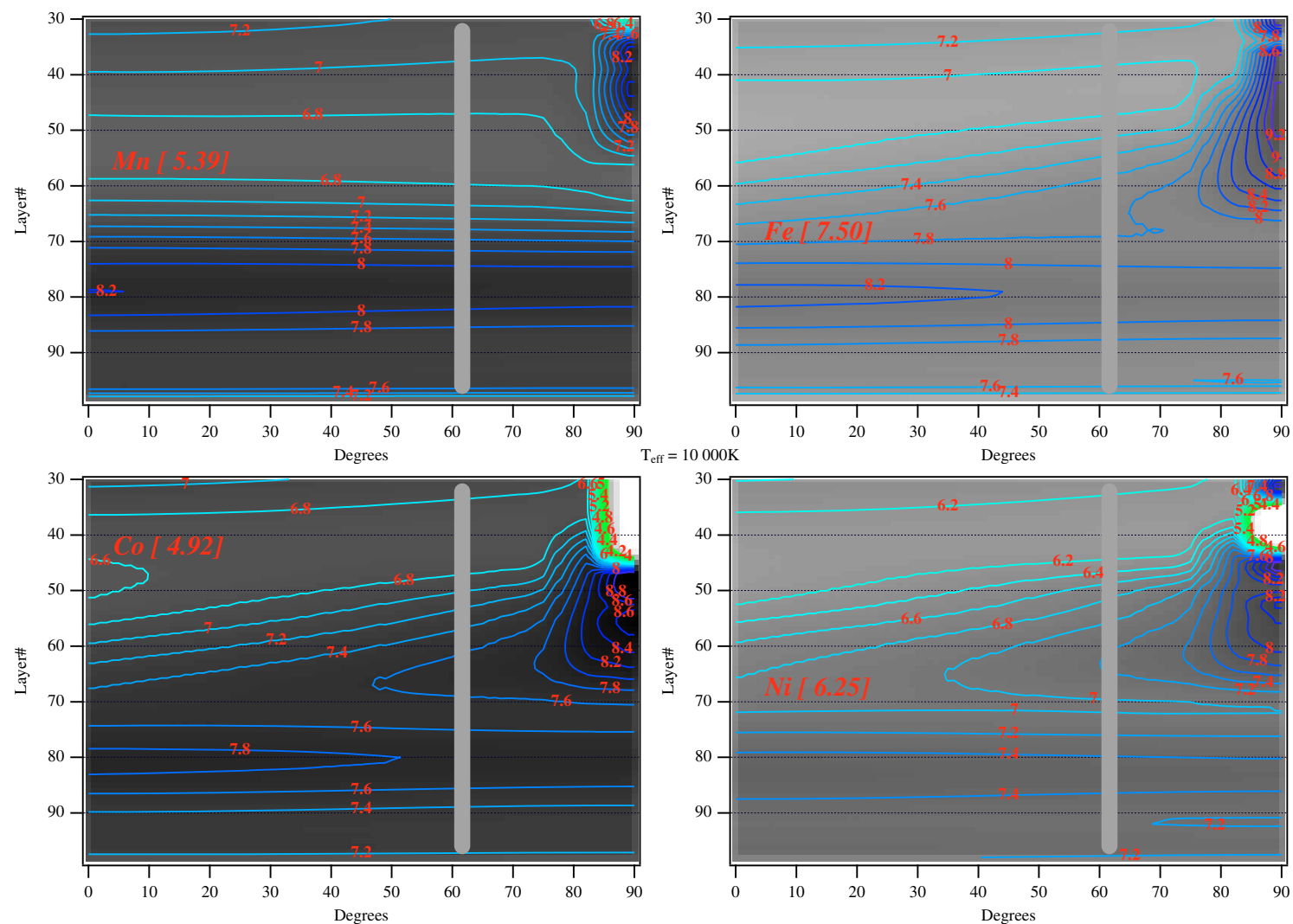

Fig. 15. Bi-dimensional stratification of $\mathrm{Mn}, \mathrm{Fe}, \mathrm{Co}$, and $\mathrm{Ni}$ for a $T_{\text {eff }}=10000 \mathrm{~K}$ model. Same annotations as in Fig. 9.
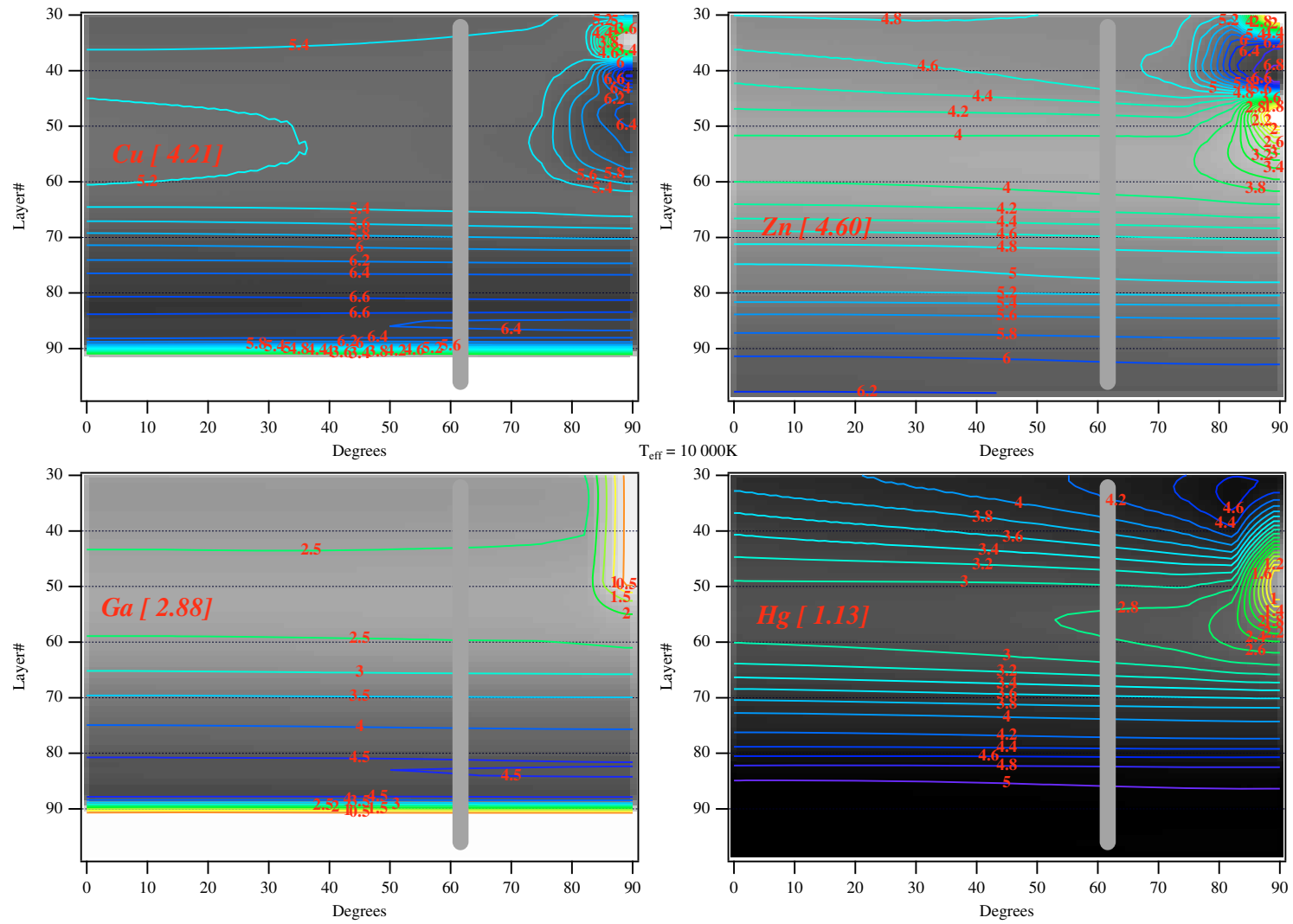

Fig. 16. Bi-dimensional stratification of $\mathrm{Cu}, \mathrm{Zn}, \mathrm{Ga}$, and $\mathrm{Hg}$ for a $T_{\text {eff }}=10000 \mathrm{~K}$ model. Same annotations as in Fig. 9. 
A\&A 516, A53 (2010)
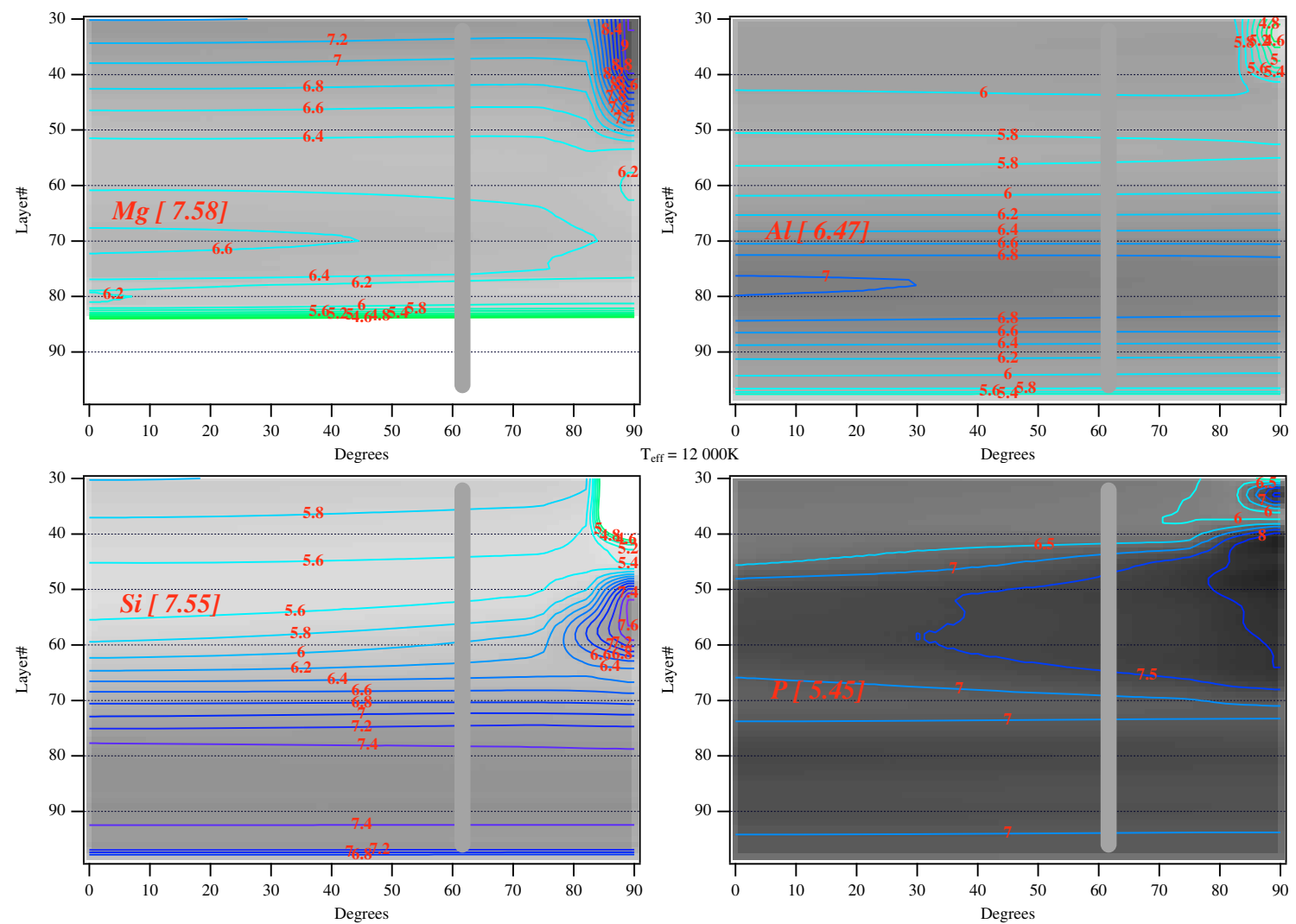

Fig. 17. Bi-dimensional stratification of $\mathrm{Mg}, \mathrm{Al}, \mathrm{Si}$, and $\mathrm{P}$ for a $T_{\text {eff }}=12000 \mathrm{~K}$ model. Same annotations as in Fig. 9.
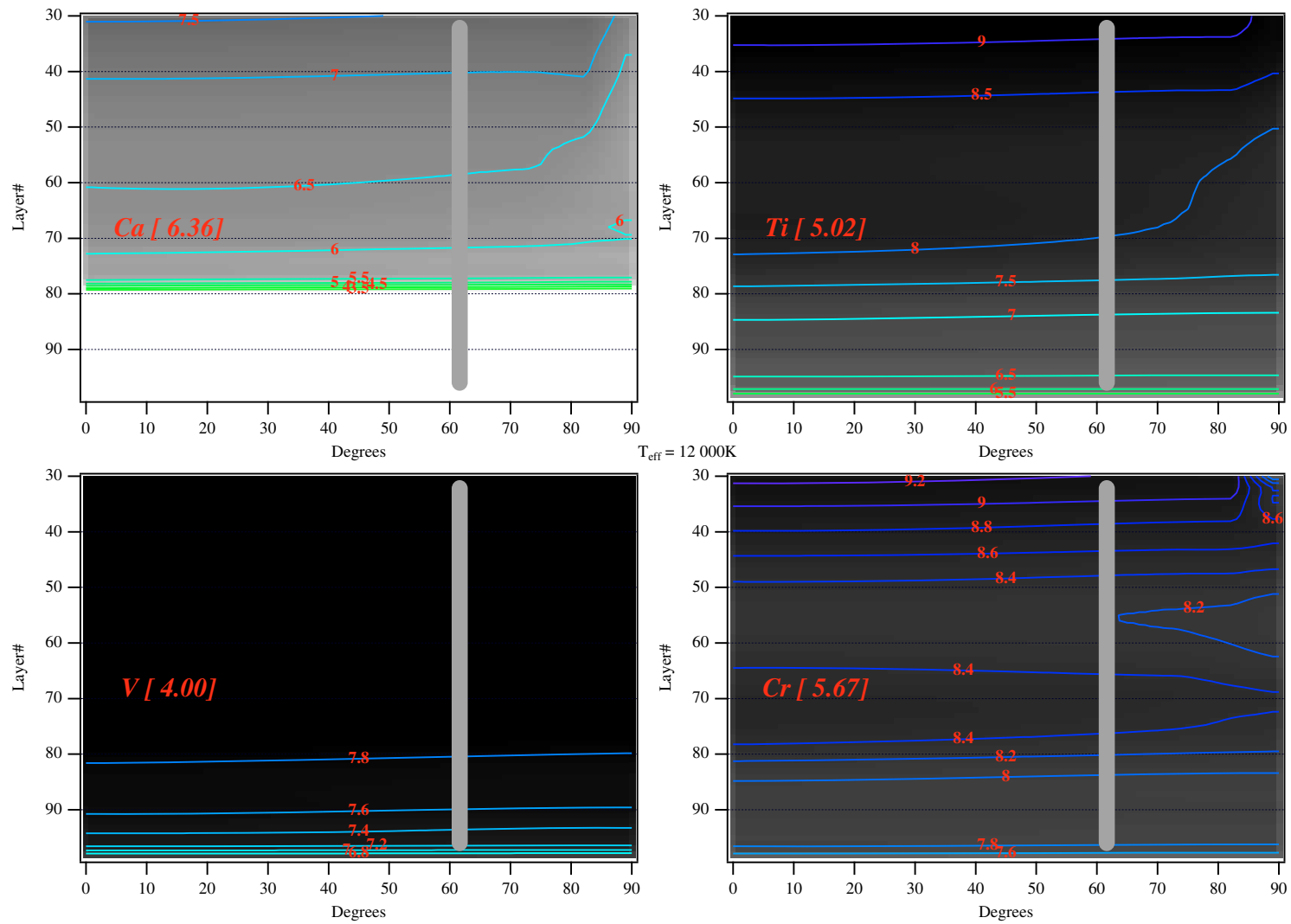

Fig. 18. Bi-dimensional stratification of $\mathrm{Ca}, \mathrm{Ti}, \mathrm{V}$, and $\mathrm{Cr}$ for a $T_{\text {eff }}=12000 \mathrm{~K}$ model. Same annotations as in Fig. 9. 
G. Alecian and M. J. Stift: Bi-dimensional stratifications in Ap stars
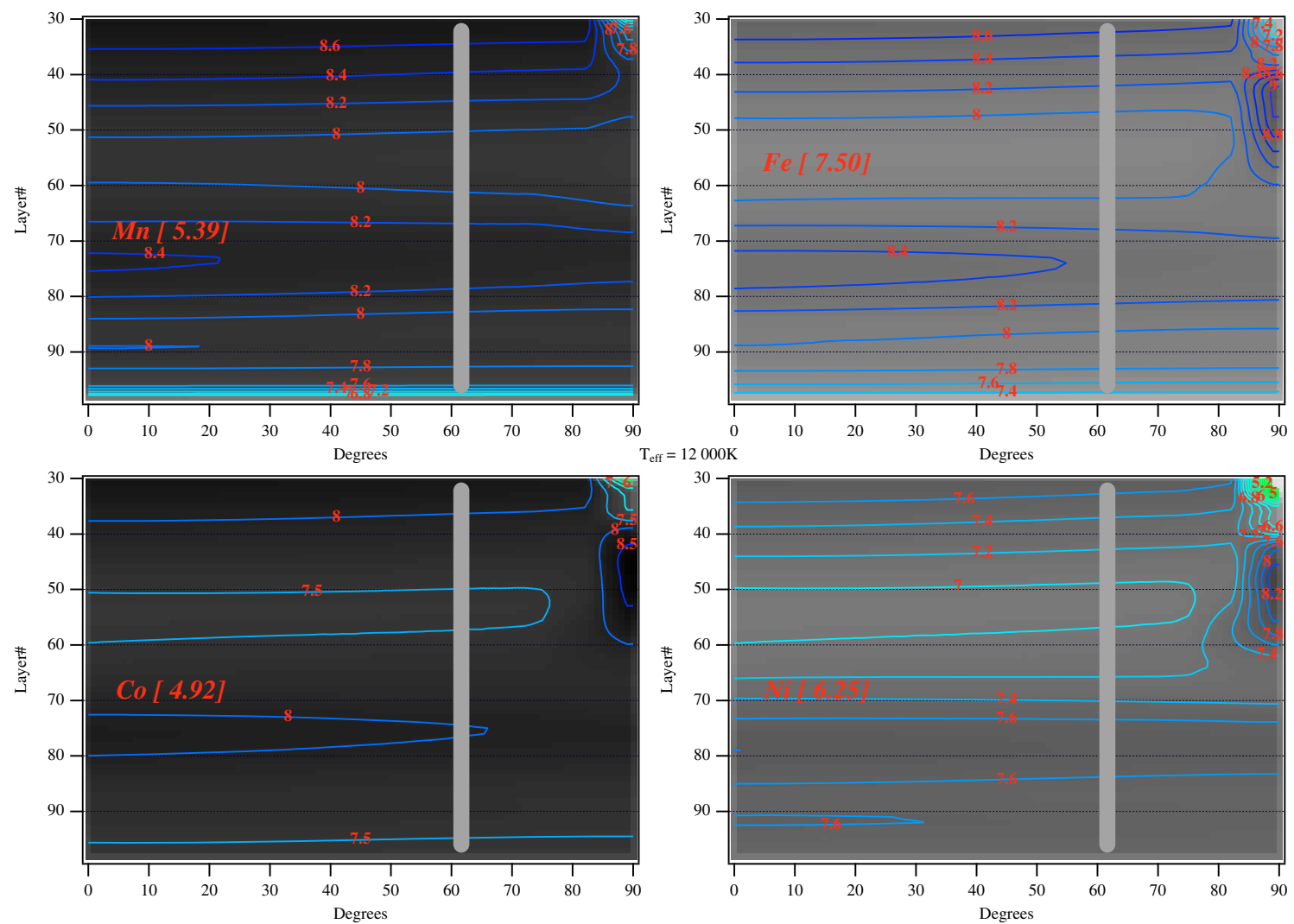

Fig. 19. Bi-dimensional stratification of $\mathrm{Mn}, \mathrm{Fe}, \mathrm{Co}$, and $\mathrm{Ni}$ for a $T_{\text {eff }}=12000 \mathrm{~K}$ model. Same annotations as in Fig. 9.
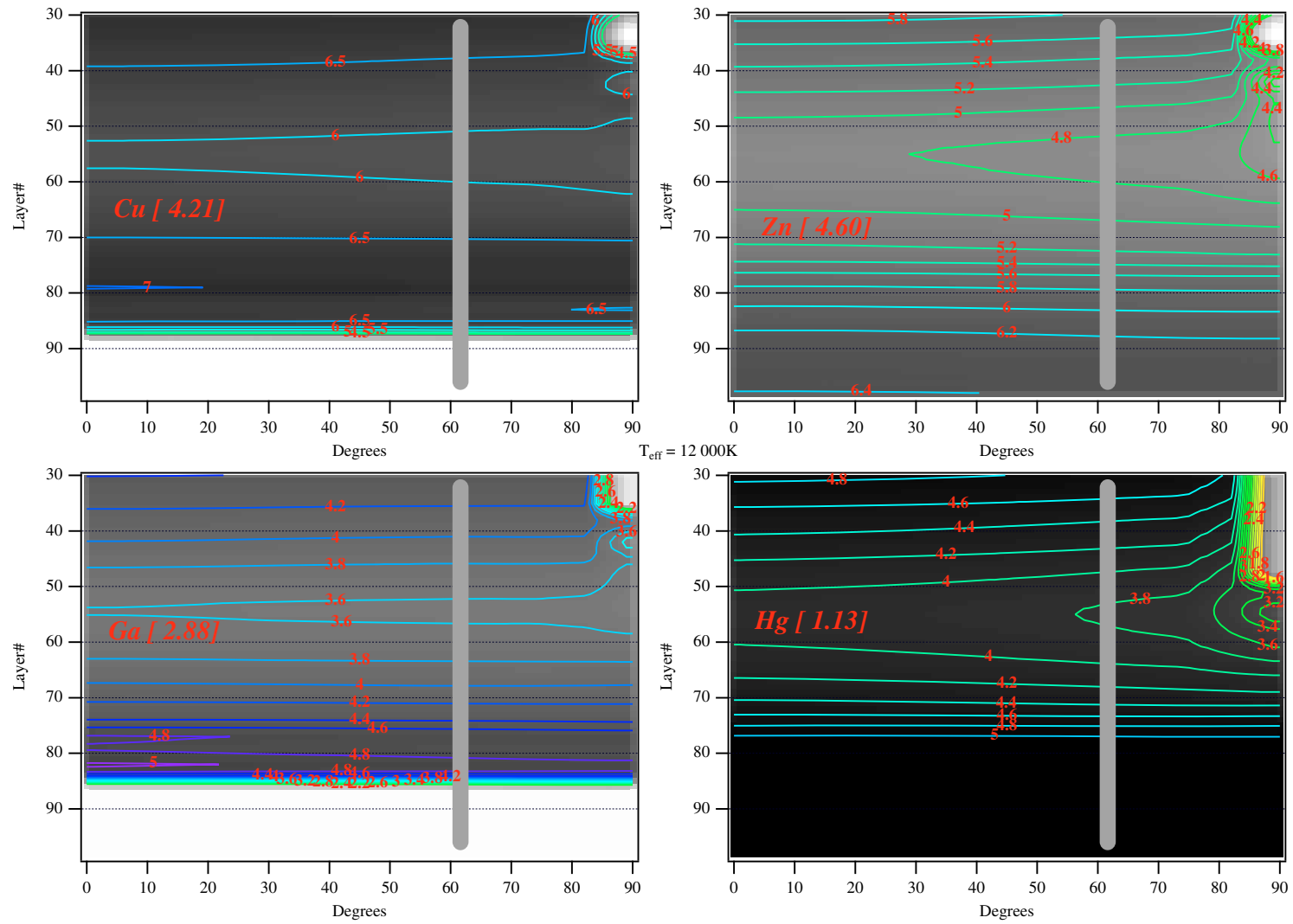

Fig. 20. Bi-dimensional stratification of $\mathrm{Cu}, \mathrm{Zn}, \mathrm{Ga}$, and $\mathrm{Hg}$ for a $T_{\text {eff }}=12000 \mathrm{~K}$ model. Same annotations as in Fig. 9. 
A\&A 516, A53 (2010)
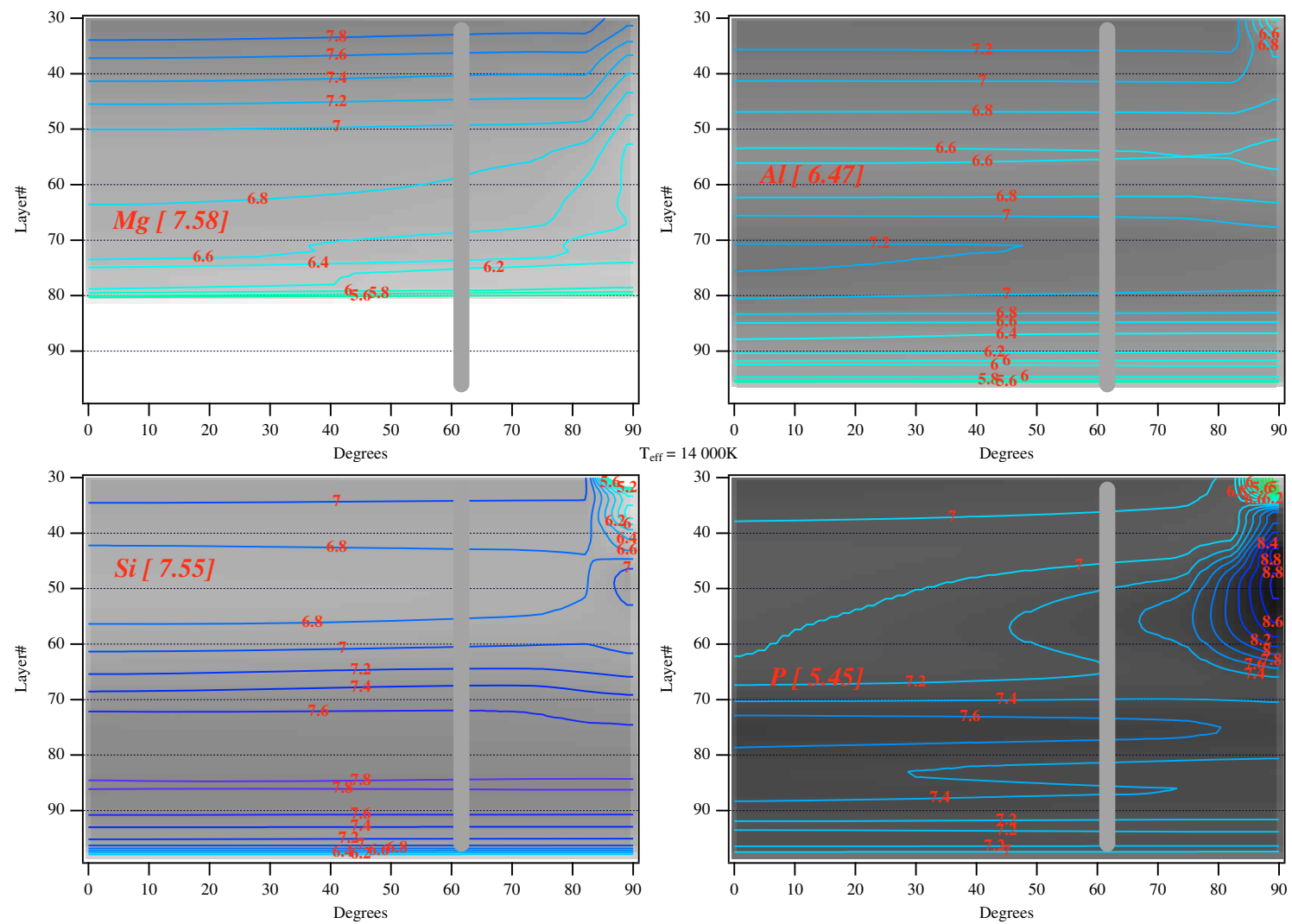

Fig. 21. Bi-dimensional stratification of $\mathrm{Mg}, \mathrm{Al}, \mathrm{Si}$, and $\mathrm{P}$ for a $T_{\text {eff }}=14000 \mathrm{~K}$ model. Same annotations as in Fig. 9.
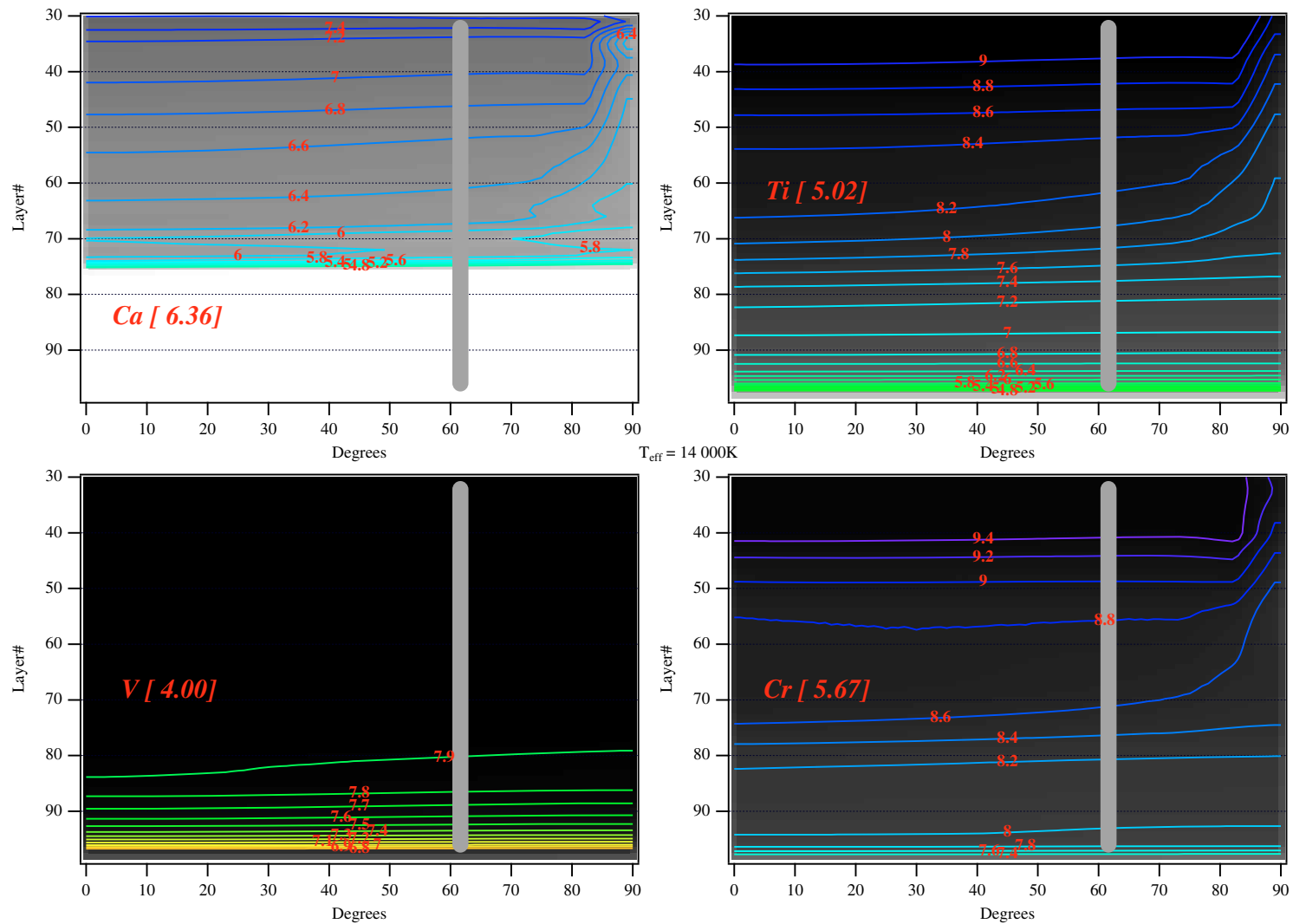

Fig. 22. Bi-dimensional stratification of $\mathrm{Ca}, \mathrm{Ti}, \mathrm{V}$, and $\mathrm{Cr}$ for a $T_{\text {eff }}=14000 \mathrm{~K}$ model. Same annotations as in Fig. 9. 
G. Alecian and M. J. Stift: Bi-dimensional stratifications in Ap stars
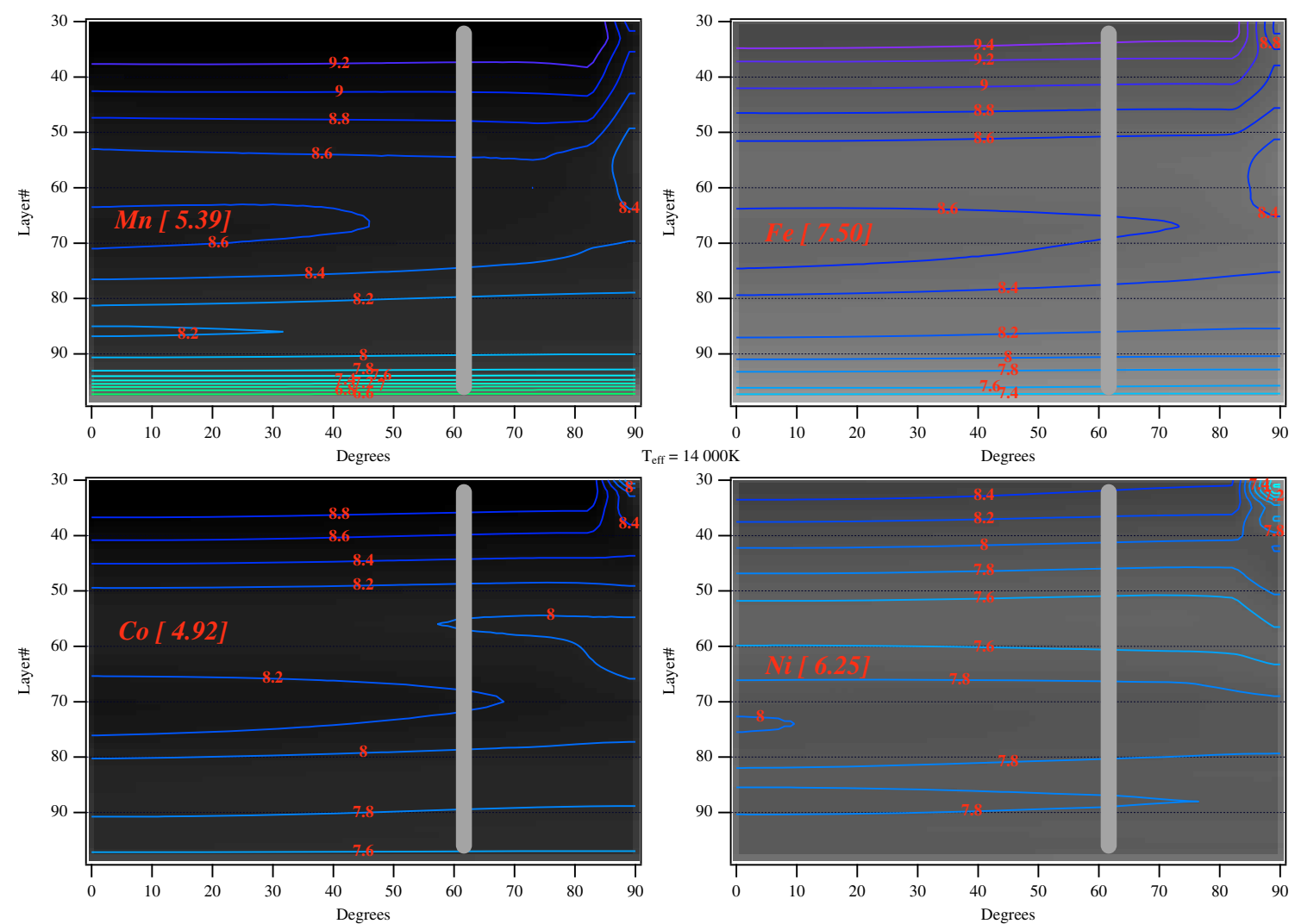

Fig. 23. Bi-dimensional stratification of $\mathrm{Mn}, \mathrm{Fe}, \mathrm{Co}$, and $\mathrm{Ni}$ for a $T_{\mathrm{eff}}=14000 \mathrm{~K}$ model. Same annotations as in Fig. 9.
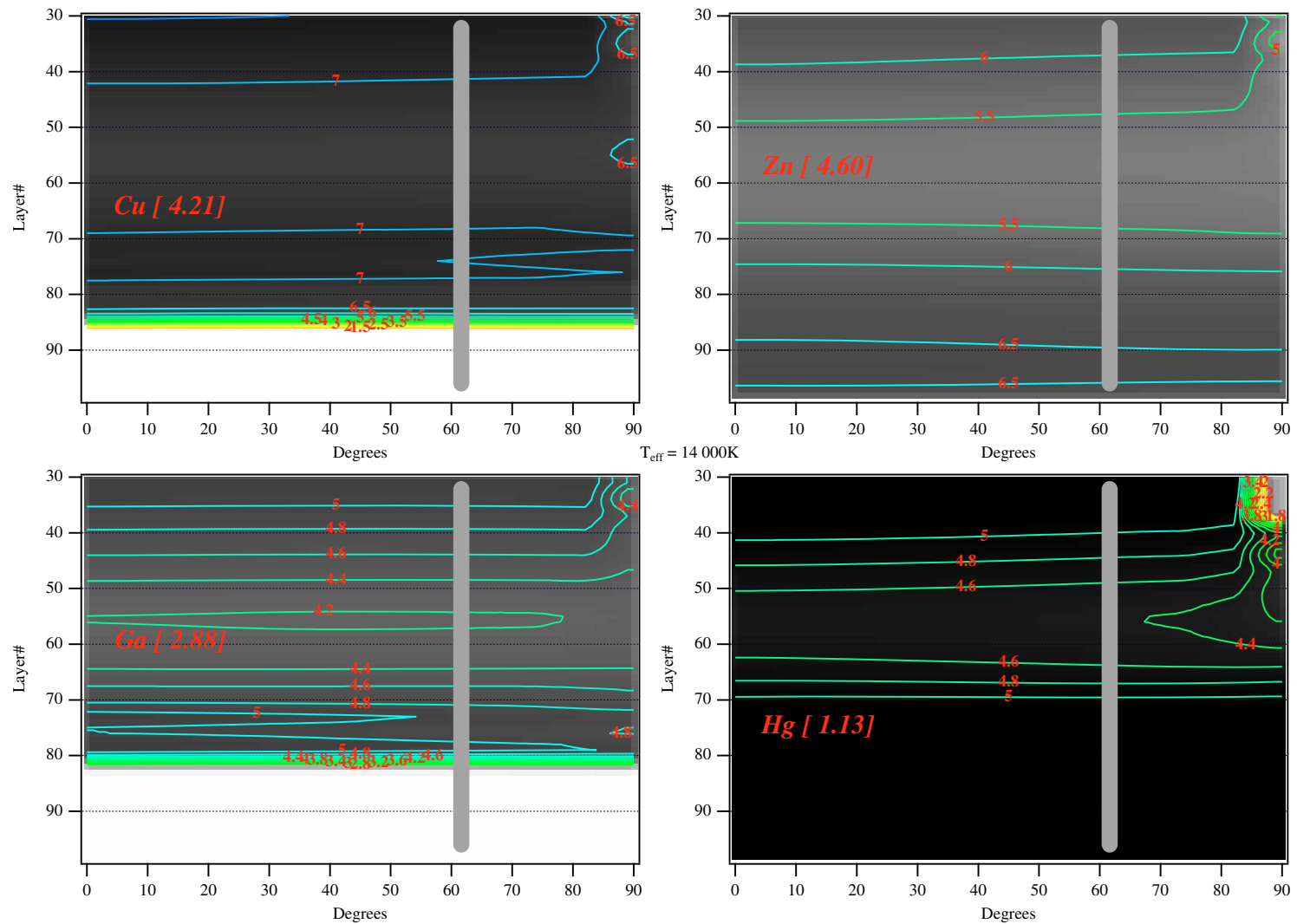

Fig. 24. Bi-dimensional stratification of $\mathrm{Cu}, \mathrm{Zn}, \mathrm{Ga}$, and $\mathrm{Hg}$ for a $T_{\text {eff }}=14000 \mathrm{~K}$ model. Same annotations as in Fig. 9. 
A\&A 516, A53 (2010)
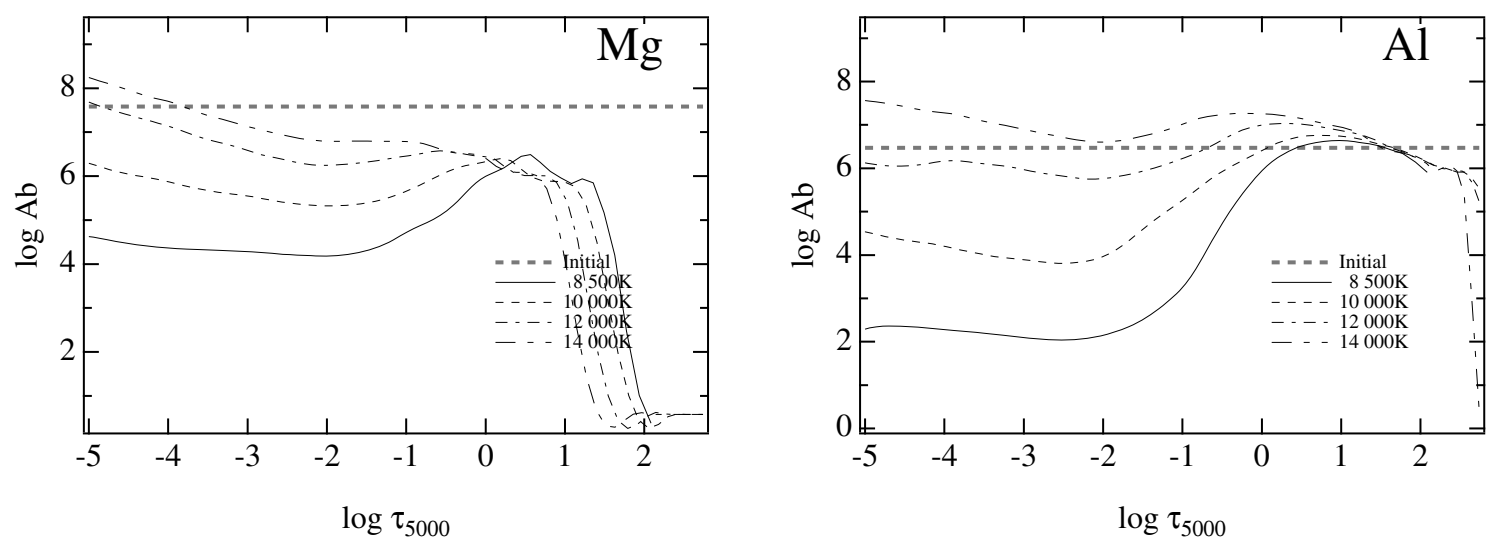

$\log \tau_{5000}$
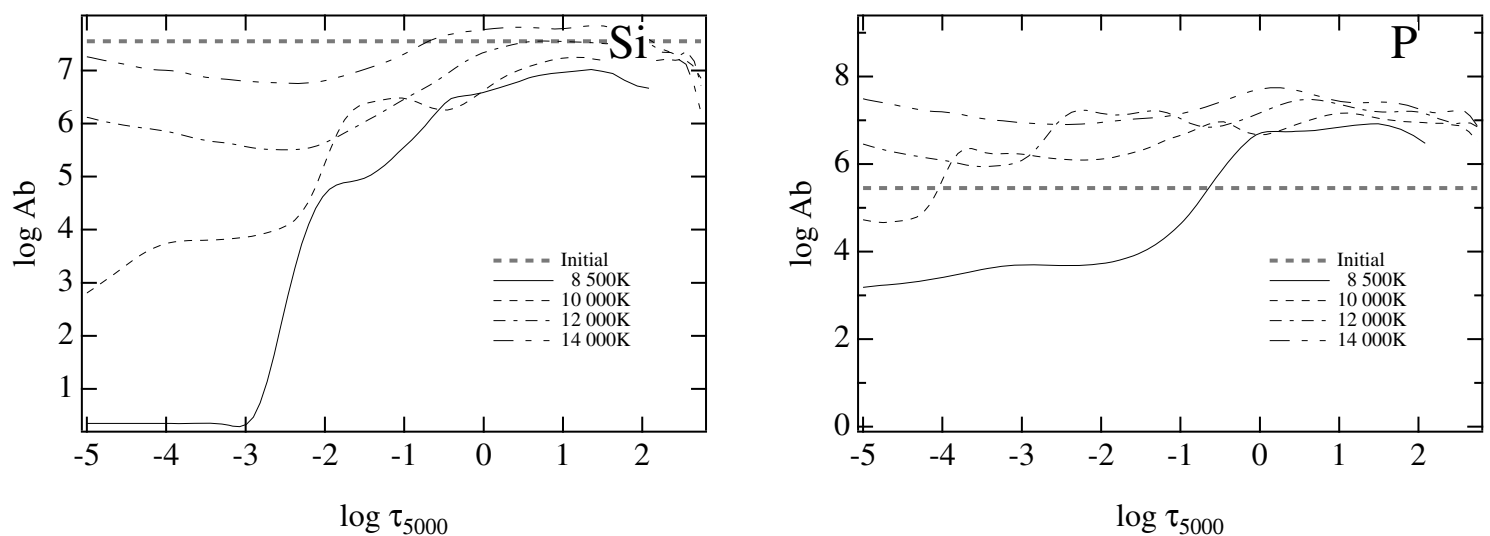

Fig. 25. 1D stratifications for the zero field case and four atmospheres with $\left(T_{\text {eff }}=8500,10000,12000,14000 \mathrm{~K}\right)$. The heavy-grey dashed line indicates the initial (solar) abundance. Case for $\mathrm{Mg}, \mathrm{Al}, \mathrm{Si}$, and $\mathrm{P}$.
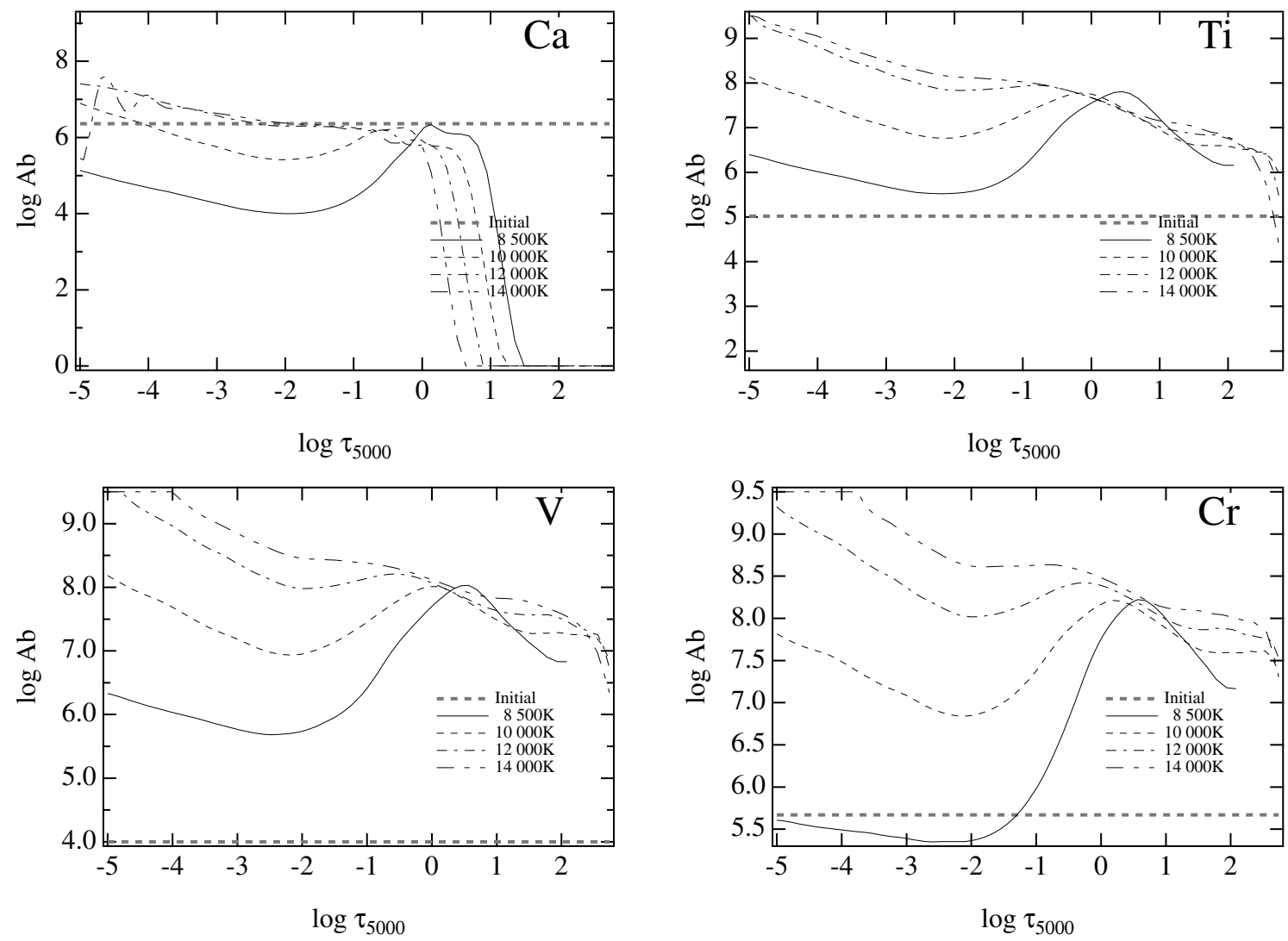

Fig. 26. Same as Fig. 25 but for $\mathrm{Ca}, \mathrm{Ti}, \mathrm{V}$, and $\mathrm{Cr}$. 
G. Alecian and M. J. Stift: Bi-dimensional stratifications in Ap stars
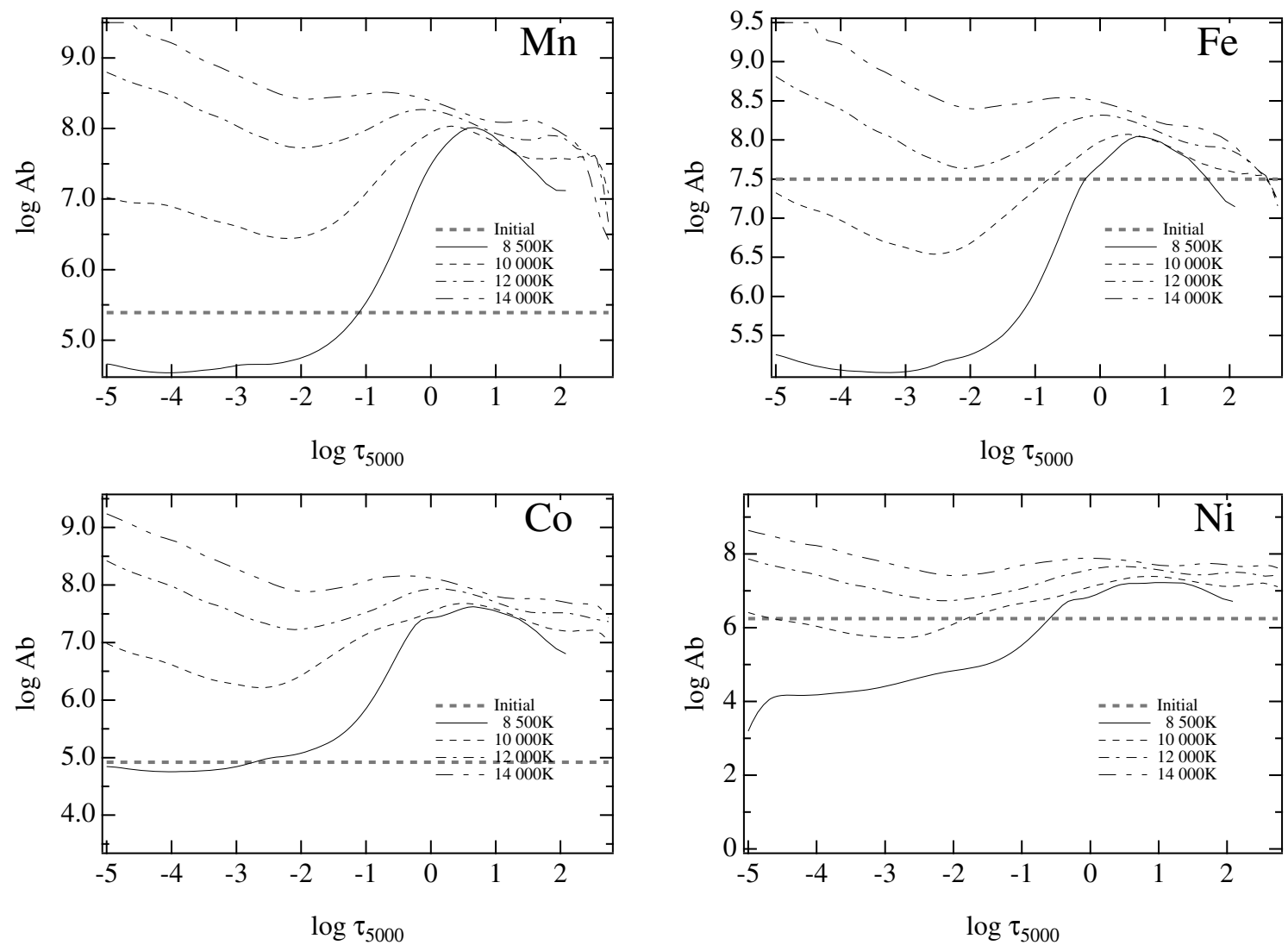

Fig. 27. Same as Fig. 25 but for Mn, Fe, Co, and Ni.
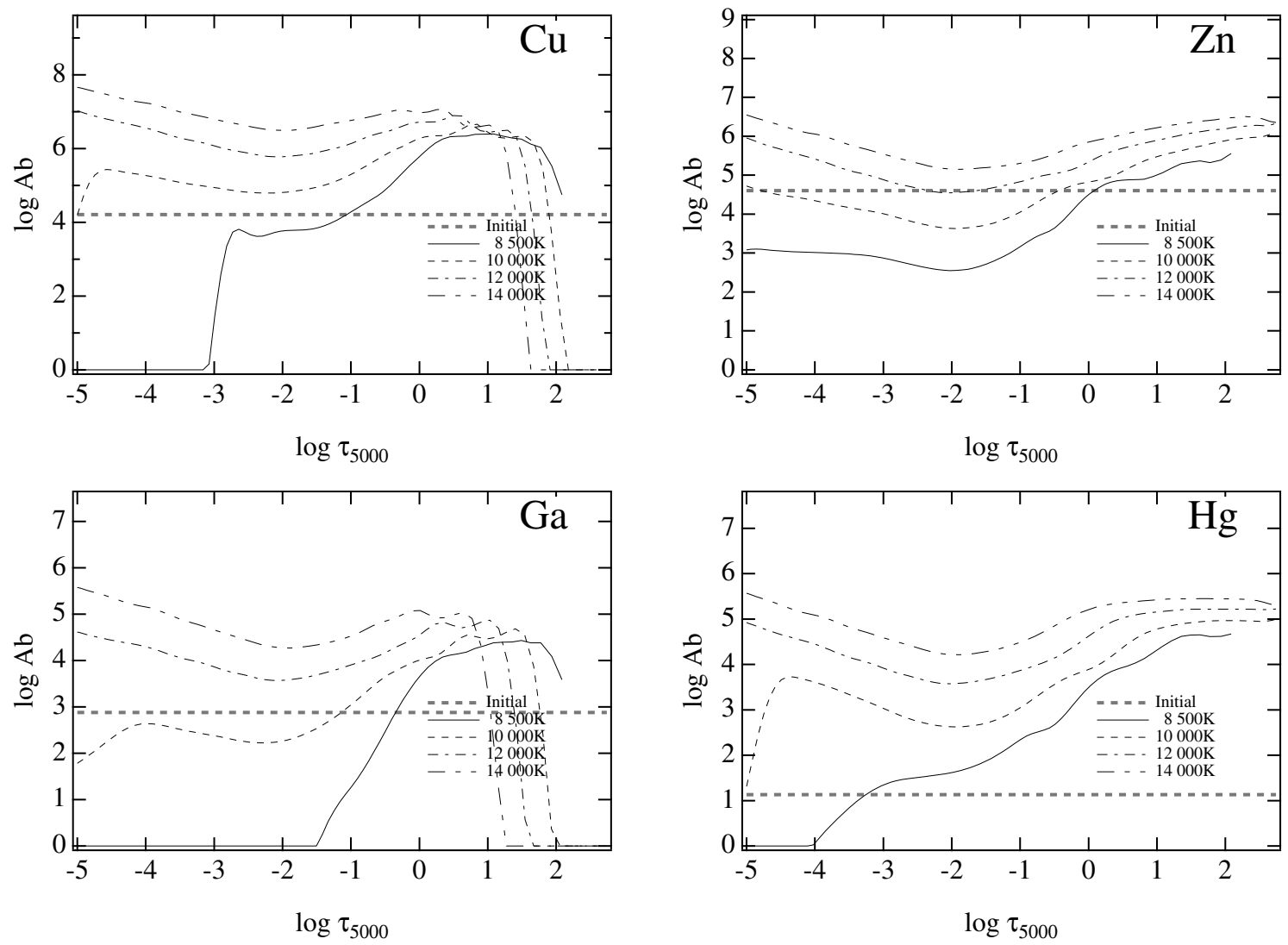

Fig. 28. Same as Fig. 25 but for $\mathrm{Cu}, \mathrm{Zn}, \mathrm{Ga}$, and $\mathrm{Hg}$. 